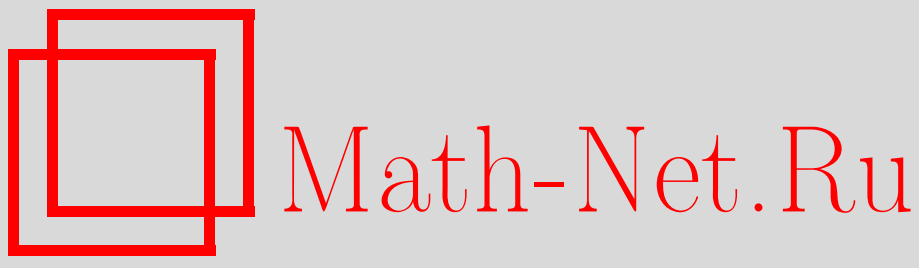

А. С. Романюк, Наилучшие $M$-членные тригонометрические приближения классов Бесова периодических функций многих переменных, Изв. РАН. Сер. матем., 2003, том 67, выпуск 2, 61-100

DOI: https://doi.org/10.4213/im427

Использование Общероссийского математического портала Math-Net.Ru подразумевает, что вы прочитали и согласны с пользовательским соглашением

http://www.mathnet.ru/rus/agreement

Параметры загрузки:

IP : 54.224 .187 .69

26 апреля 2023 г., 15:23:09 
УДК 517.5

\author{
А. C. Романюк
}

\title{
Наилучшие $M$-членные тригонометрические приближения классов Бесова периодических функций многих переменных
}

\begin{abstract}
Получены точные по порядку оценки наилучших $M$-членных тригонометрических приближений классов Бесова $B_{p, \theta}^{r}$ периодических функций многих переменных в пространстве $L_{q}$ для некоторых соотношений между параметрами $p$ и $q$.

Библиография: 38 наименований.
\end{abstract}

\section{Введение}

В настоящей работе изучаются вопросы наилучших $M$-членных тригонометрических приближений классов периодических функций многих переменных $B_{p, \theta}^{r}$ в пространстве $L_{q}$. Введем некоторые обозначения и определения.

Пусть $\mathbb{R}^{d}, d \geqslant 1,-$ евклидово пространство с элементами $x=\left(x_{1}, \ldots, x_{d}\right)$ и $(x, y)=x_{1} y_{1}+\cdots+x_{d} y_{d} ; L_{p}\left(\pi_{d}\right), \pi_{d}=\prod_{j=1}^{d}[-\pi, \pi]$, обозначает множество функций $f(x), 2 \pi$-периодических по каждой переменной и таких, что

$$
\begin{aligned}
\|f\|_{p}= & \left((2 \pi)^{-d} \int_{\pi_{d}}|f(x)|^{p} d x\right)^{1 / p}<\infty, \quad 1 \leqslant p<\infty, \\
& \|f\|_{\infty}=\operatorname{vraisup}_{x \in \pi_{d}}|f(x)|<\infty, \quad p=\infty .
\end{aligned}
$$

Далее всюду предполагаем, что для $f \in L_{p}\left(\pi_{d}\right)$ выполнено дополнительное условие

$$
\int_{-\pi}^{\pi} f(x) d x_{j}=0, \quad j=\overline{1, d} .
$$

Множество таких функций будем обозначать $L_{p}^{0}\left(\pi_{d}\right)$.

Для векторов $k=\left(k_{1}, \ldots, k_{d}\right), k_{j} \in \mathbb{Z}$, и $s=\left(s_{1}, \ldots, s_{d}\right), s_{j} \in \mathbb{N}, j=\overline{1, d}$, положим

$$
\rho(s)=\left\{k: 2^{s_{j}-1} \leqslant\left|k_{j}\right|<2^{s_{j}}\right\}
$$

и для $f \in L_{p}^{0}\left(\pi_{d}\right)$ обозначим

$$
\delta_{s}(f, x)=\sum_{k \in \rho(s)} \hat{f}(k) e^{i(k, x)},
$$

где $\hat{f}(k)=(2 \pi)^{-d} \int_{\pi_{d}} f(t) e^{-i(k, t)} d t-$ коэффициенты Фурье функции $f(x)$.

(C) А.С. РОмАнюк, 2003 
Пусть $1<p<\infty, r=\left(r_{1}, \ldots, r_{d}\right), r_{j}>0, j=\overline{1, d}$. Тогда классы $B_{p, \theta}^{r}$, введенные О. В. Бесовым [1], можно определить следующим образом (см., например, [2]):

$$
B_{p, \theta}^{r}=\left\{f(x) \mid\|f\|_{B_{p, \theta}^{r}}=\left(\sum_{s} 2^{(s, r) \theta}\left\|\delta_{s}(f, x)\right\|_{p}^{\theta}\right)^{1 / \theta} \leqslant 1\right\}
$$

при $1 \leqslant \theta<\infty$ и

$$
B_{p, \infty}^{r}=\left\{f(x) \mid\|f\|_{B_{p, \infty}^{r}}=\sup _{s} 2^{(s, r)}\left\|\delta_{s}(f, x)\right\|_{p} \leqslant 1\right\} .
$$

Обратим внимание, что приведенное определение классов $B_{p, \theta}^{r}$ можно распространить и на крайние значения $p=1, \infty$, несколько видоизменив при этом "блоки" $\delta_{s}(f, x)$.

Именно, пусть $V_{l}(t), l \in \mathbb{N}$, обозначает ядро Валле-Пуссена вида

$$
V_{l}(t)=1+2 \sum_{k=1}^{l} \cos k t+2 \sum_{k=l+1}^{2 l}\left(1-\frac{k-l}{l}\right) \cos k t .
$$

Сопоставим каждому вектору $s=\left(s_{1}, \ldots, s_{d}\right), s_{j} \in \mathbb{N}, j=\overline{1, d}$, полином

$$
A_{s}(x)=\prod_{j=1}^{d}\left(V_{2^{s_{j}}}\left(x_{j}\right)-V_{2^{s_{j}-1}}\left(x_{j}\right)\right)
$$

и для $f \in L_{p}^{0}\left(\pi_{d}\right), \quad 1 \leqslant p \leqslant \infty$, положим $A_{s}(f, x)=f(x) * A_{s}(x)$, где знак “*” обозначает операцию свертки. Тогда при каждом $1 \leqslant p \leqslant \infty, r=\left(r_{1}, \ldots, r_{d}\right)$, $r_{j}>0, j=\overline{1, d}$, классы $B_{p, \theta}^{r}$ определяются следуюшим образом:

$$
B_{p, \theta}^{r}=\left\{f(x) \mid\|f\|_{B_{p, \theta}^{r}}=\left(\sum_{s} 2^{(s, r) \theta}\left\|A_{s}(f, x)\right\|_{p}^{\theta}\right)^{1 / \theta} \leqslant 1\right\}
$$

при $1 \leqslant \theta<\infty$ и

$$
B_{p, \infty}^{r}=\left\{f(x) \mid\|f\|_{B_{p, \infty}^{r}}=\sup _{s} 2^{(s, r)}\left\|A_{s}(f, x)\right\|_{p} \leqslant 1\right\} .
$$

Отметим, что классы $B_{p, \infty}^{r}$ совпадают с классами $H_{p}^{r}[3$, с. 31$]$, которые, в свою очередь, являются аналогами известных классов, введенных С. М. Никольским (см. $\left[4\right.$, с. 189]). Напомним определение классов $H_{p}^{r}$, определив предварительно классы $H_{p, l}^{r}, l \in \mathbb{N}[3$, с. 31$]$.

Класс $H_{p, l}^{r}$ - это множество функций $f(x) \in L_{p}^{0}\left(\pi_{d}\right)$ таких, что

$$
\left\|\Delta_{t}^{l} f(x)\right\|_{p} \leqslant \prod_{j=1}^{d}\left|t_{j}\right|^{r_{j}}, \quad l>\max _{j} r_{j}
$$


где $t=\left(t_{1}, \ldots, t_{d}\right), \Delta_{t}^{l} f(x)$ - смешанная $l$-я разность с шагом $t_{j}$ по каждой переменной $x_{j}, j=\overline{1, d}$. Из теоремы, доказанной В. Н. Темляковым (см. [3, теорема 1.1]), следует, что классы $H_{p, l}^{r}$ с различными $l$ эквивалентны. Поскольку всюду ниже речь будет идти только о порядковых соотношениях, и с этой точки зрения классы $H_{p, l}^{r}$ с различными $l>\max _{j} r_{j}$ неразличимы, то в обозначениях $H_{p, l}^{r}$ индекс $l$ опускается.

Напомним также и определение классов $W_{p, \alpha}^{r}$ (см., например, [3, с. 31]). Пусть $F_{r}(x, \alpha), r=\left(r_{1}, \ldots, r_{d}\right),-$ многомерные аналоги ядер Бернулли, т.е.

$$
F_{r}(x, \alpha)=2^{d} \sum_{k} \prod_{j=1}^{d} k_{j}^{-r_{j}} \cos \left(k_{j} x_{j}-\frac{\alpha_{j} \pi}{2}\right), \quad r_{j}>0, \quad \alpha_{j} \in \mathbb{R},
$$

и в сумме участвуют только те векторы $k=\left(k_{1}, \ldots, k_{d}\right)$, для которых $k_{j}>0$, $j=\overline{1, d}$. Обозначим через $W_{p, \alpha}^{r}$ класс функций $f(x)$, представимых в виде

$$
f(x)=\varphi(x) * F_{r}(x, \alpha)=(2 \pi)^{-d} \int_{\pi_{d}} \varphi(y) F_{r}(x-y, \alpha) d y,
$$

где $\varphi \in L_{p}\left(\pi_{d}\right),\|\varphi\|_{p} \leqslant 1$.

Далее, не умаляя обшности, будем считать, что координаты вектора $r=\left(r_{1}, \ldots\right.$ $\ldots, r_{d}$ ) упорядочены в следуюшем виде: $0<r_{1}=r_{2}=\cdots=r_{\nu}<r_{\nu+1} \leqslant \cdots \leqslant r_{d}$. Вектору $r=\left(r_{1}, \ldots, r_{d}\right)$ сопоставим вектор $\gamma=\left(\gamma_{1}, \ldots, \gamma_{d}\right), \gamma_{j}=r_{j} / r_{1}, j=\overline{1, d}$, которому, в свою очередь, сопоставляется вектор $\gamma^{\prime}=\left(\gamma_{1}^{\prime}, \ldots, \gamma_{d}^{\prime}\right)$, где $\gamma_{j}=\gamma_{j}^{\prime}$ при $j=\overline{1, \nu}_{\text {и }} 1<\gamma_{j}^{\prime}<\gamma_{j}$ при $j=\overline{\nu+1, d}$.

Если $A$ - конечное множество, то через $|A|$ будем обозначать количество его элементов.

Приводимые ниже результаты будем формулировать в терминах порядковых соотношений. При этом функции $\mu_{1}(N)$ и $\mu_{2}(N)$ называем функциями одного порядка и пишем $\mu_{1} \asymp \mu_{2}$, если сушествуют постоянные $C_{1} \leqslant C_{2}$ такие, что $C_{1} \mu_{2}(N) \leqslant$ $\mu_{1}(N) \leqslant C_{2} \mu_{2}(N)$. Аналогично определяются порядковые неравенства $\mu_{1} \ll \mu_{2}$ и $\mu_{1} \gg \mu_{2}$. Все постоянные $C_{i}, i=1,2, \ldots$, которые будут встречаться в работе, могут зависеть только от тех параметров, которые входят в определение классов, а также метрик, в которых измеряется погрешность приближения и размерности пространства $\mathbb{R}^{d}$.

Теперь приведем определение величины, которая будет нами исследоваться.

Для $f \in L_{q}\left(\pi_{d}\right)$ обозначим

$$
e_{M}(f)_{q}=\inf _{k^{j}, c_{j}}\left\|f(\cdot)-\sum_{j=1}^{M} c_{j} e^{i\left(k^{j}, \cdot\right)}\right\|_{q}
$$

где $\left\{k^{j}\right\}_{j=1}^{M}$ - система векторов $k^{j}=\left(k_{1}^{j}, \ldots, k_{d}^{j}\right)$ с целочисленньги координатами, $c_{j}$ - произвольные коэффициенты. Если $F$ - некоторый функциональный класс, то полагаем

$$
e_{M}(F)_{q}=\sup _{f \in F} e_{M}(f)_{q}
$$


Величина $e_{M}(f)_{2}$ для функций одной переменной была введена С. Б. Стечкиным [5] при формулировке критерия абсолютной сходимости рядов Фурье. Несколько позже величины $e_{M}(f)_{q}$ и $e_{M}(F)_{q}, 1 \leqslant q \leqslant \infty$, начали исследовать уже с точки зрения аппроксимации (см., например, [6]-[12]). Следует отметить, что полученные при этом результаты нашли применение в вопросах исследования других аппроксимативных характеристик, в частности колмогоровских поперечников соответствующих классов функций ${ }^{1}$.

Систематическое изучение величин $e_{M}(F)_{q}$ на классах периодических функций многих переменных $W_{p, \alpha}^{r}$ и $H_{p}^{r}$ было положено В. Н. Темляковым [13]. Важно отметить, что при изучении им величины $e_{M}\left(W_{p, \alpha}^{r}\right)_{q}, 1<p<q \leqslant 2$, $r_{1}>2\left(\frac{1}{p}-\frac{1}{q}\right)$, было обнаружено, что наилучшие $M$-членные тригонометрические приближения убывают при $M \rightarrow \infty$ существенно быстрее колмогоровских поперечников $d_{M}\left(W_{p, \alpha}^{r}, L_{q}\right)$.

Затем исследования величин $e_{M}(F)_{q}$ на тех же классах функций $W_{p, \alpha}^{r}$ и $H_{p}^{r}$ были продолжены Э. С. Белинским [14]-[17]. Об этом более подробно будет идти речь в комментариях к полученным нами результатам. Отметим еше, что в последнее время исследование наилучших $M$-членных приближений тех или иных функциональных классов получило мощное развитие в работах многих авторов [18]-[26]. Там же можно ознакомиться и с более подробной библиографией по данному вопросу.

Цель настоящей работы - продолжить исследования в указанном направлении на классах $B_{p, \theta}^{r}$. Отметим, что некоторая часть из приведенных ниже результатов была ранее опубликована с доказательством, либо анонсирована в [27]-[29]. Поскольку за последнее время методы нахождения оценок наилучших $M$-членных приближений получили определенные усовершенствования, то в настояшей работе приводятся модифицированные доказательства и ранее полученные атвором результаты.

\section{§ 1. Вспомогательные утверждения}

Напомним несколько известных утверждений, которые будут систематически использоваться на протяжении всей работы.

Теорема А (Литтлвуда-Пэли [4, с. 65]). Пусть задано $1<p<\infty . \quad C y$ ществуют положительные числа $C_{1}$ и $C_{2}$ такие, что для каждой функиии $f \in L_{p}^{0}\left(\pi_{d}\right)$ справедлива оценка

$$
C_{1}\|f\|_{p} \leqslant\left\|\left\{\sum_{s}\left|\delta_{s}(f, x)\right|^{2}\right\}^{1 / 2}\right\|_{p} \leqslant C_{2}\|f\|_{p}
$$

\footnotetext{
${ }^{1}$-мерным колмогоровским поперечником центрально-симметричного множества $\Phi$ банахова пространства $\mathscr{X}$ называется величина

$$
d_{n}(\Phi, \mathscr{X})=\inf _{L_{n}} \sup _{f \in \Phi} \inf _{a \in L_{n}}\|f-a\| \mathscr{X}
$$
}

где $L_{n}$ - подпространство размерности $n$ пространства $\mathscr{X}$. 
Лемма А [3, с. 25]. Пусть $1 \leqslant p<q<\infty u f \in L_{p}^{0}\left(\pi_{d}\right)$. Тогда

$$
\|f\|_{q} \ll\left\{\sum_{s}\left(\left\|\delta_{s}(f, x)\right\|_{p} 2^{\|s\|_{1}\left(\frac{1}{p}-\frac{1}{q}\right)}\right)^{q}\right\}^{\frac{1}{q}}
$$

$\|s\|_{1}=s_{1}+s_{2}+\cdots+s_{d}$.

Лемма Б [3, с. 11]. Имеет место оценка

$$
\sum_{\left(s, \gamma^{\prime}\right) \geqslant n} 2^{-\beta(s, \gamma)} \asymp 2^{-\beta n} n^{\nu-1}, \quad \beta>0 .
$$

Лемма В [3, с. 11]. Пусть $\gamma=\left(\gamma_{1}, \ldots, \gamma_{d}\right)$ u $\delta=\left(\delta_{1}, \ldots, \delta_{d}\right)$ таковы, ито $1=\gamma_{1}=\delta_{1}=\cdots=\gamma_{\nu}=\delta_{\nu} u 1<\delta_{j}<\gamma_{j}, j=\overline{\nu+1, d}$. Тогда

$$
\sum_{(s, \gamma) \leqslant n} 2^{\beta(s, \delta)} \ll 2^{\beta n} n^{\nu-1}, \quad \beta>0
$$

ЛЕмма $\Gamma\left[3\right.$, с. 28]. Пусть $1<p<q \leqslant \infty u f \in L_{p}^{0}\left(\pi_{d}\right)$. Тогда

$$
\|f\|_{p} \gg\left\{\sum_{s}\left(\left\|\delta_{s}(f, x)\right\|_{q} 2^{\|s\|_{1}\left(\frac{1}{q}-\frac{1}{p}\right)}\right)^{p}\right\}^{\frac{1}{p}}
$$

Теорема Б [30]. Пусть $n=\left(n_{1}, \ldots, n_{d}\right), \quad n_{j}$ - челье неотричательные иисла, $j=\overline{1, d}$, и $t(x)=\sum_{\left|k_{j}\right| \leqslant n_{j}} c_{k} e^{i(k, x)}$. Тогда при $1 \leqslant q<p \leqslant \infty$ имеет место неравенство

$$
\|t\|_{p} \leqslant 2^{d} \prod_{j=1}^{d} n_{j}^{\left(\frac{1}{q}-\frac{1}{p}\right)}\|t\|_{q} .
$$

Это неравенство было установлено С. М. Никольским и получило название неравенство разных метрик. В случае $d=1$ и $p=\infty$ соответствуюшее неравенство доказал Джексон [31].

Теорема В [3, с. 32]. Для того чтобь $f \in H_{p}^{r}, \quad 1 \leqslant p \leqslant \infty$, необходимо $и$ достаточно, чтобы выполнялись соотношения

$$
\begin{aligned}
\left\|\delta_{s}(f, x)\right\|_{p} \ll 2^{-(s, r)}, & 1<p<\infty \\
\left\|A_{s}(f, x)\right\|_{p} \ll 2^{-(s, r)}, & 1 \leqslant p \leqslant \infty .
\end{aligned}
$$




\section{$\S 2$. Порядки величин $e_{M}\left(B_{p, \theta}^{r}\right)_{q}$ при $1 \leqslant p \leqslant 2<q<\infty$ и $2 \leqslant p<q<\infty$}

В этом параграфе рассмотрим один из тех случаев $(1 \leqslant p \leqslant 2<q<\infty)$, при исследовании которого проявилось так называемое явление "малой гладкости". В связи с этим обстоятельством возникла необходимость рассмотреть несколько соотношений между параметрами $r_{1}, p$ и $q$, в каждом из которых требовалось несколько модифицировать метод получения оценок сверху.

Отметим, что явление “малой гладкости” впервые было обнаружено Б. С. Кашиным [32] в процессе установления оценок колмогоровских поперечников классов Соболева $W_{1}^{r}$ функций одной переменной в пространстве $L_{q}$. Иными словами, в [32] было выявлено, что поведение поперечников $d_{M}\left(W_{1}^{r}, L_{q}\right), 2<q<\infty$, в случае $1-\frac{1}{q}<r<1$ сушественно отличается от случая $r>1$. Затем подобного рода явления были обнаружены в некоторых случаях и при исследовании аппроксимативных характеристик классов периодических функций многих переменных.

В нашей ситуации явление "малой гладкости" состоит в том, что при переходе показателя гладкости $r$ класса $B_{p, \theta}^{r}$ через значение $r_{1}=\frac{1}{p}$ происходит "скачок" в оценке величины $e_{M}\left(B_{p, \theta}^{r}\right)_{q}$. Иными словами, оценку $e_{M}\left(B_{p, \theta}^{r}\right)_{q}$ при $r_{1}=\frac{1}{p}$ нельзя получить предельным переходом из оценок $e_{M}\left(B_{p, \theta}^{r}\right)_{q}$ при $\frac{1}{p}-\frac{1}{q}<r_{1}<\frac{1}{p}$, либо при $r_{1}>\frac{1}{p}$.

Имеет место следуюшее утверждение.

Tеорема 2.1. Пусть $1 \leqslant p \leqslant 2<q<\infty, 1 \leqslant \theta \leqslant \infty$. Тогда

$$
e_{M}\left(B_{p, \theta}^{r}\right)_{q} \asymp \begin{cases}M^{-\frac{1}{2}}\left(\log ^{\nu} M\right)^{\frac{1}{\theta^{\prime}}}, & r_{1}=\frac{1}{p}, \\ \left(M^{-1} \log ^{\nu-1} M\right)^{r_{1}-\frac{1}{p}+\frac{1}{2}}\left(\log ^{\nu-1} M\right)^{\frac{1}{2}-\frac{1}{\theta},} & r_{1}>\frac{1}{p}, \\ M^{-\frac{q}{2}\left(r_{1}-\frac{1}{p}+\frac{1}{q}\right)}\left(\log ^{\nu-1} M\right)^{(q-1)\left(r_{1}-\frac{1}{p}+\frac{q^{\prime}}{q \theta^{\prime}}\right)_{+}}, & \frac{1}{p}-\frac{1}{q}<r_{1}<\frac{1}{p},\end{cases}
$$

əде $a_{+}=\max \{a, 0\}, \quad \frac{1}{b}+\frac{1}{b^{\prime}}=1$.

ДокАЗАТЕЛЬСтво. Сначала установим оценку сверху в случае $r_{1} \geqslant \frac{1}{p}$. Для этого нам понадобится вспомогательное утверждение. Пусть $\Omega_{L}-$ множество, содержащее не более чем $L$ векторов $k=\left(k_{1}, \ldots, k_{d}\right)$ с целочисленными координатами, а $P\left(\Omega_{L}, x\right)$ - произвольный тригонометрический полином, состоящий из гармоник с "номерами" из $\Omega_{L}$. Справедлива

Лемма 2.1 [16]. Пусть $2<q<\infty$. Тогда для всякого тригонометрического полинома $P\left(\Omega_{N}, x\right)$ и для любого $M<N$ найдется тригонометрический полином $P\left(\Omega_{M}, x\right)$, для которого имеет место оценка

$$
\left\|P\left(\Omega_{N}, x\right)-P\left(\Omega_{M}, x\right)\right\|_{q} \leqslant C_{1} \sqrt{N M^{-1}}\left\|P\left(\Omega_{N}, x\right)\right\|_{2},
$$

причем $\Omega_{M} \subset \Omega_{N}$.

Итак, пусть $f(x)$ - некоторая функция из класса $B_{p, \theta}^{r}, \quad M$ - произвольное натуральное число и $n$ подобрано из соотношения $M \asymp 2^{n} n^{\nu-1}$. Приближающий 
полином $P\left(\Omega_{M}, x\right)$, доставляющий для $f(x)$ требуемую оценку приближения, будем подбирать в виде

$$
P\left(\Omega_{M}, x\right)=\sum_{\left(s, \gamma^{\prime}\right)<n} \delta_{s}(f, x)+\sum_{n \leqslant\left(s, \gamma^{\prime}\right)<\alpha n} P\left(\Omega_{N_{s}}, x\right),
$$

где $P\left(\Omega_{N_{s}}, x\right)$ - полиномы, которые будут построены для каждого "блока" $\delta_{s}(f, x)$ согласно лемме 2.1 , а число $\alpha>1$ будет подбираться в процессе построения.

Предположим, что искомый полином построен. Тогда согласно $(2.2)$ и в силу теоремы А и неравенства Минковского будем иметь

$$
\begin{aligned}
\left\|f(x)-P\left(\Omega_{M}, x\right)\right\|_{q} \ll & \left\|\left(\sum_{n \leqslant\left(s, \gamma^{\prime}\right)<\alpha n}\left|\delta_{s}(f, x)-P\left(\Omega_{N_{s}}, x\right)\right|^{2}\right)^{1 / 2}\right\|_{q} \\
& +\left\|\sum_{\left(s, \gamma^{\prime}\right) \geqslant \alpha n} \delta_{s}(f, x)\right\|_{q}=I_{1}+I_{2} .
\end{aligned}
$$

Оценим сначала слагаемое $I_{2}$. Пусть $q_{0}$ - некоторое число, удовлетворяющее условию $p<q_{0}<q$. Тогда, принимая во внимание, что $\left\|\delta_{s}(f, x)\right\|_{q_{0}} \asymp\left\|A_{s}(f, x)\right\|_{q_{0}}$, $1<q_{0}<\infty$, и воспользовавшись леммой $\mathrm{A}$, получим

$$
\begin{aligned}
I_{2} & =\left\|\sum_{\left(s, \gamma^{\prime}\right) \geqslant \alpha n} \delta_{s}(f, x)\right\|_{q} \ll\left\{\sum_{\left(s, \gamma^{\prime}\right) \geqslant \alpha n}\left(\left\|\delta_{s}(f, x)\right\|_{q_{0}} 2^{\|s\|_{1}\left(\frac{1}{q_{0}}-\frac{1}{q}\right)}\right)^{q}\right\}^{\frac{1}{q}} \\
& \asymp\left\{\sum_{\left(s, \gamma^{\prime}\right) \geqslant \alpha n}\left(\left\|A_{s}(f, x)\right\|_{q_{0}} 2^{\|s\|_{1}\left(\frac{1}{q_{0}}-\frac{1}{q}\right)}\right)^{q}\right\}^{\frac{1}{q}} .
\end{aligned}
$$

Далее, применив к $A_{s}(f, x)$ как к полиному степени $2^{s_{j}}$ по переменной $x_{j}$ неравенство (1.5), продолжим оценку:

$$
\begin{aligned}
I_{2} & \ll\left\{\sum_{\left(s, \gamma^{\prime}\right) \geqslant \alpha n}\left(\left\|A_{s}(f, x)\right\|_{p} 2^{\|s\|_{1}\left(\frac{1}{p}-\frac{1}{q_{0}}\right)} 2^{\|s\|_{1}\left(\frac{1}{q_{0}}-\frac{1}{q}\right)}\right)^{q}\right\}^{\frac{1}{q}} \\
& =\left\{\sum_{\left(s, \gamma^{\prime}\right) \geqslant \alpha n}\left(\left\|A_{s}(f, x)\right\|_{p} 2^{\|s\|_{1}\left(\frac{1}{p}-\frac{1}{q}\right)}\right)^{q}\right\}^{\frac{1}{q}}=I_{3} .
\end{aligned}
$$

Для последующей оценки $I_{3}$ нам понадобится рассмотреть несколько случаев.

а) Пусть $1 \leqslant \theta \leqslant q$. Тогда в силу неравенства [33, с. 43]

$$
\left(\sum_{k}\left|a_{k}\right|^{\nu_{2}}\right)^{\frac{1}{\nu_{2}}} \leqslant\left(\sum_{k}\left|a_{k}\right|^{\nu_{1}}\right)^{\frac{1}{\nu_{1}}}, \quad 1 \leqslant \nu_{1} \leqslant \nu_{2}<\infty
$$


имеем

$$
\begin{aligned}
I_{3} & \leqslant\left(\sum_{\left(s, \gamma^{\prime}\right) \geqslant \alpha n}\left\|A_{s}(f, x)\right\|_{p}^{\theta} 2^{\|s\|_{1}\left(\frac{1}{p}-\frac{1}{q}\right) \theta}\right)^{\frac{1}{\theta}} \\
& \leqslant\left(\sum_{\left(s, \gamma^{\prime}\right) \geqslant \alpha n} 2^{(s, r) \theta}\left\|A_{s}(f, x)\right\|_{p}^{\theta} 2^{-\left(s, \gamma^{\prime}\right)\left(r_{1}-\frac{1}{p}+\frac{1}{q}\right) \theta}\right)^{\frac{1}{\theta}} \\
& \leqslant 2^{-\alpha n\left(r_{1}-\frac{1}{p}+\frac{1}{q}\right)}\left(\sum_{\left(s, \gamma^{\prime}\right) \geqslant \alpha n} 2^{(s, r) \theta}\left\|A_{s}(f, x)\right\|_{p}^{\theta}\right)^{\frac{1}{\theta}} \\
& \leqslant 2^{-\alpha n\left(r_{1}-\frac{1}{p}+\frac{1}{q}\right)}\|f\|_{B_{p, \theta}^{r}} \leqslant 2^{-\alpha n\left(r_{1}-\frac{1}{p}+\frac{1}{q}\right)} .
\end{aligned}
$$

б) Пусть $q<\theta<\infty$, и пусть

$$
I_{3}=\left\{\sum_{\left(s, \gamma^{\prime}\right) \geqslant \alpha n} 2^{q(s, r)}\left\|A_{s}(f, x)\right\|_{p}^{q} 2^{-q\left((s, r)-\|s\|_{1}\left(\frac{1}{p}-\frac{1}{q}\right)\right)}\right\}^{\frac{1}{q}} .
$$

Применив к (2.7) неравенство Гёльдера с показателем $\frac{\theta}{q}$ и воспользовавшись оценкой (1.3), получим

$$
\begin{aligned}
I_{3} & \leqslant\left(\sum_{\left(s, \gamma^{\prime}\right) \geqslant \alpha n} 2^{(s, r) \theta}\left\|A_{s}(f, x)\right\|_{p}^{\theta}\right)^{\frac{1}{\theta}}\left(\sum_{\left(s, \gamma^{\prime}\right) \geqslant \alpha n} 2^{-q\left((s, r)-\|s\|_{1}\left(\frac{1}{p}-\frac{1}{q}\right)\right) \frac{\theta}{\theta-q}}\right)^{\frac{1}{q}-\frac{1}{\theta}} \\
& \leqslant\|f\|_{B_{p, \theta}^{r}}\left(\sum_{\left(s, \gamma^{\prime}\right) \geqslant \alpha n} 2^{-q\left((s, r)-\|s\|_{1}\left(\frac{1}{p}-\frac{1}{q}\right)\right) \frac{\theta}{\theta-q}}\right)^{\frac{1}{q}-\frac{1}{\theta}} \\
& \leqslant\left(\sum_{\left(s, \gamma^{\prime}\right) \geqslant \alpha n} 2^{-q\left(r_{1}-\frac{1}{p}+\frac{1}{q}\right) \frac{\theta}{\theta-q}(s, \gamma)}\right)^{\frac{1}{q}-\frac{1}{\theta}} \asymp 2^{-\alpha n\left(r_{1}-\frac{1}{p}+\frac{1}{q}\right)} n^{(\nu-1)\left(\frac{1}{q}-\frac{1}{\theta}\right)} .
\end{aligned}
$$

в) Пусть $\theta=\infty$. Тогда, принимая во внимание, что в этом случае $B_{p, \infty}^{r}=H_{p}^{r}$, и в силу теоремы В $\left\|A_{s}(f, x)\right\|_{p} \ll 2^{-(s, r)}$, из (2.7) будем иметь

$$
\begin{aligned}
I_{3} & \ll\left(\sum_{\left(s, \gamma^{\prime}\right) \geqslant \alpha n} 2^{-q\left((s, r)-\|s\|_{1}\left(\frac{1}{p}-\frac{1}{q}\right)\right)}\right)^{\frac{1}{q}} \leqslant\left(\sum_{\left(s, \gamma^{\prime}\right) \geqslant \alpha n} 2^{-q(s, \gamma)\left(r_{1}-\frac{1}{p}+\frac{1}{q}\right)}\right)^{\frac{1}{q}} \\
& \asymp 2^{-\alpha n\left(r_{1}-\frac{1}{p}+\frac{1}{q}\right)} n^{(\nu-1) \frac{1}{q}} .
\end{aligned}
$$

Таким образом, подставив полученные оценки (2.6), (2.8) и (2.9) в (2.4), приходим к оценке

$$
I_{2} \ll 2^{-\alpha n\left(r_{1}-\frac{1}{p}+\frac{1}{q}\right)} n^{(\nu-1)\left(\frac{1}{q}-\frac{1}{\theta}\right)_{+}} .
$$


Теперь перейдем к оценке $I_{1}$. Заметим, что поскольку $q \in(2, \infty)$, то

$$
\begin{aligned}
I_{1} & =\left\|\left(\sum_{n \leqslant\left(s, \gamma^{\prime}\right)<\alpha n}\left|\delta_{s}(f, x)-P\left(\Omega_{N_{s}}, x\right)\right|^{2}\right)^{\frac{1}{2}}\right\|_{q} \\
& =\left(\left\|\sum_{n \leqslant\left(s, \gamma^{\prime}\right)<\alpha n}\left|\delta_{s}(f, x)-P\left(\Omega_{N_{s}}, x\right)\right|^{2}\right\|_{q / 2}\right)^{\frac{1}{2}} \\
& \leqslant\left(\sum_{n \leqslant\left(s, \gamma^{\prime}\right)<\alpha n}\left\|\left|\delta_{s}(f, x)-P\left(\Omega_{N_{s}}, x\right)\right|^{2}\right\|_{q / 2}\right)^{\frac{1}{2}} \\
& =\left(\sum_{n \leqslant\left(s, \gamma^{\prime}\right)<\alpha n}\left\|\delta_{s}(f, x)-P\left(\Omega_{N_{s}}, x\right)\right\|_{q}^{2}\right)^{\frac{1}{2}} .
\end{aligned}
$$

Далее, воспользовавшись сначала леммой 2.1, а затем оценкой $(1.5)$, получим

$$
\begin{aligned}
I_{1} & \ll\left(\sum_{n \leqslant\left(s, \gamma^{\prime}\right)<\alpha n} N_{s}^{-1} 2^{\|s\|_{1}}\left\|\delta_{s}(f, x)\right\|_{2}^{2}\right)^{\frac{1}{2}} \\
& \asymp\left(\sum_{n \leqslant\left(s, \gamma^{\prime}\right)<\alpha n} N_{s}^{-1} 2^{\|s\|_{1}}\left\|A_{s}(f, x)\right\|_{2}^{2}\right)^{\frac{1}{2}} \\
& \ll\left(\sum_{n \leqslant\left(s, \gamma^{\prime}\right)<\alpha n} N_{s}^{-1} 2^{\|s\|_{1}} 2^{2\left(\frac{1}{p}-\frac{1}{2}\right)\|s\|_{1}}\left\|A_{s}(f, x)\right\|_{p}^{2}\right)^{\frac{1}{2}} \\
& =\left(\sum_{n \leqslant\left(s, \gamma^{\prime}\right)<\alpha n} N_{s}^{-1} 2^{\frac{2\|s\|_{1}}{p}}\left\|A_{s}(f, x)\right\|_{p}^{2}\right)^{\frac{1}{2}} .
\end{aligned}
$$

Чтобы продолжить оценку (2.11), нам понадобится теперь рассмотреть отдельно случаи $r_{1}=\frac{1}{p}$ и $r_{1}>\frac{1}{p}$.

Итак, пусть $r_{1}=\frac{1}{p}$. Тогда зададим число $\alpha$ равенством

$$
\alpha=\frac{q}{2}\left(1+\frac{(\nu-1) \log n}{n}\right),
$$

а каждому вектору $s=\left(s_{1}, \ldots, s_{d}\right)$, удовлетворяющему условию $n \leqslant\left(s, \gamma^{\prime}\right)<\alpha n$, поставим в соответствие число

$$
N_{s}=\left[2^{n} n^{\frac{\nu}{\theta}-1}\left\|A_{s}(f, x)\right\|_{p} 2^{r_{1}\|s\|_{1}}\right]+1, \quad 1 \leqslant \theta<\infty
$$

(соответственно, $N_{s}=\left[2^{n} n^{-1}\left\|A_{s}(f, x)\right\|_{p} 2^{r_{1}\|s\|_{1}}\right]+1$ при $\left.\theta=\infty\right)$, где $[a]-$ целая часть числа $a$. 
Нетрудно показать, что при таком выборе чисел $N_{s}$ выполнено соотношение $\sum_{n \leqslant\left(s, \gamma^{\prime}\right)<\alpha n} N_{s} \ll M$. Действительно, если $1 \leqslant \theta<\infty$, то согласно неравенству Гёльдера (с естественной модификацией при $\theta=1$ ) будем иметь

$$
\begin{aligned}
\sum_{n \leqslant\left(s, \gamma^{\prime}\right)<\alpha n} N_{s} \ll & n^{d}+2^{n} n^{\frac{\nu}{\theta}-1} \sum_{n \leqslant\left(s, \gamma^{\prime}\right)<\alpha n} 2^{r_{1}\|s\|_{1}}\left\|A_{s}(f, x)\right\|_{p} \\
= & n^{d}+2^{n} n^{\frac{\nu}{\theta}-1} \sum_{n \leqslant\left(s, \gamma^{\prime}\right)<\alpha n} 2^{(s, r)}\left\|A_{s}(f, x)\right\|_{p} 2^{-(s, r)} 2^{r_{1}\|s\|_{1}} \\
\leqslant & n^{d}+2^{n} n^{\frac{\nu}{\theta}-1}\left(\sum_{n \leqslant\left(s, \gamma^{\prime}\right)<\alpha n} 2^{(s, r) \theta}\left\|A_{s}(f, x)\right\|_{p}^{\theta}\right)^{\frac{1}{\theta}} \\
& \times\left(\sum_{n \leqslant\left(s, \gamma^{\prime}\right)<\alpha n} 2^{r_{1}\|s\|_{1} \theta^{\prime}} 2^{-(s, r) \theta^{\prime}}\right)^{\frac{1}{\theta^{\prime}}} \\
\ll & n^{d}+2^{n} n^{\frac{\nu}{\theta}-1}\|f\|_{B_{p, \theta}^{r}}\left(\sum_{n \leqslant\left(s, \gamma^{\prime}\right)<\alpha n} 2^{r_{1}\left(s, \gamma^{\prime}\right) \theta^{\prime}} 2^{-r_{1}(s, \gamma) \theta^{\prime}}\right)^{\frac{1}{\theta^{\prime}}} \\
\ll & n^{d}+2^{n} n^{\frac{\nu}{\theta}-1}\left(\sum_{l=n}^{[\alpha n]+1} 2^{r_{1} l \theta^{\prime}} \sum_{l \leqslant\left(s, \gamma^{\prime}\right)<l+1} 2^{-r_{1}(s, \gamma) \theta^{\prime}}\right)^{\frac{1}{\theta^{\prime}}} \cdot
\end{aligned}
$$

Применив к последней сумме в (2.12) соотношение (1.3), получим требуемую оценку

$$
\begin{aligned}
\sum_{n \leqslant\left(s, \gamma^{\prime}\right)<\alpha n} N_{s} & \ll n^{d}+2^{n} n^{\frac{\nu}{\theta}-1}\left(\sum_{l=n}^{[\alpha n]+1} 2^{r_{1} l \theta^{\prime}} 2^{-r_{1} l \theta^{\prime}} l^{\nu-1}\right)^{\frac{1}{\theta^{\prime}}} \\
& =n^{d}+2^{n} n^{\frac{\nu}{\theta}-1}\left(\sum_{l=n}^{[\alpha n]+1} l^{\nu-1}\right)^{\frac{1}{\theta^{\prime}}} \ll n^{d}+2^{n} n^{\frac{\nu}{\theta}-1} n^{\frac{\nu}{\theta^{\prime}}} \\
& =n^{d}+2^{n} n^{\nu-1} \ll 2^{n} n^{\nu-1} \asymp M .
\end{aligned}
$$

В случае $\theta=\infty$ для $f \in B_{p, \infty}^{r}$ согласно теореме В $\left\|A_{s}(f, x)\right\|_{p} \ll 2^{-(s, r)}$ и, следовательно, для количества гармоник будем иметь оценку

$$
\begin{aligned}
\sum_{n \leqslant\left(s, \gamma^{\prime}\right)<\alpha n} N_{s} & \ll n^{d}+2^{n} n^{-1} \sum_{n \leqslant\left(s, \gamma^{\prime}\right)<\alpha n} 2^{r_{1}\|s\|_{1}}\left\|A_{s}(f, x)\right\|_{p} \\
& \ll n^{d}+2^{n} n^{-1} \sum_{n \leqslant\left(s, \gamma^{\prime}\right)<\alpha n} 2^{r_{1}\|s\|_{1} 2^{-(s, r)}} \\
& =n^{d}+2^{n} n^{-1} \sum_{n \leqslant\left(s, \gamma^{\prime}\right)<\alpha n} 2^{r_{1}\|s\|_{1}} 2^{-r_{1}(s, \gamma)} \\
& \ll n^{d}+2^{n} n^{-1} \sum_{l=n}^{[\alpha n]+1} 2^{r_{1} l} \sum_{l \leqslant\left(s, \gamma^{\prime}\right)<l+1} 2^{-r_{1}(s, \gamma)} \\
& \ll n^{d}+2^{n} n^{-1} \sum_{l=n}^{[\alpha n]+1} 2^{r_{1} l} 2^{-r_{1} l} l^{\nu-1} \ll n^{d}+2^{n} n^{-1} n^{\nu} \\
& \ll 2^{n} n^{\nu-1} \asymp M
\end{aligned}
$$


Прежде чем продолжить оценку (2.11), заметим следующее. Может случиться, что $N_{s} \geqslant 2^{\|s\|_{1}}$ для некоторых векторов $s=\left(s_{1}, \ldots, s_{d}\right)$; тогда в роли полиномов $P\left(\Omega_{N_{s}}, x\right)$ будем выбирать соответствуюший “блок" $\delta_{s}(f, x)$. Это замечание будем учитывать далее и при рассмотрении других случаев.

Таким образом, подставляя значения $N_{s}$ в $(2.11)$ и учитывая, что $r_{1}=\frac{1}{p}$, получим

$$
\begin{aligned}
I_{1} & \ll 2^{-\frac{n}{2}} n^{-\frac{1}{2}\left(\frac{\nu}{\theta}-1\right)}\left(\sum_{n \leqslant\left(s, \gamma^{\prime}\right)<\alpha n} 2^{\|s\|_{1}\left(\frac{2}{p}-r_{1}\right)}\left\|A_{s}(f, x)\right\|_{p}\right)^{\frac{1}{2}} \\
& =2^{-\frac{n}{2}} n^{-\frac{1}{2}\left(\frac{\nu}{\theta}-1\right)}\left(\sum_{n \leqslant\left(s, \gamma^{\prime}\right)<\alpha n} 2^{r_{1}\|s\|_{1}}\left\|A_{s}(f, x)\right\|_{p}\right)^{\frac{1}{2}} .
\end{aligned}
$$

Далее, повторив те же выкладки, что и при проведении оценок (2.12), (2.13) и $\left(2.13^{\prime}\right)$, приходим к соотношению

$$
I_{1} \ll 2^{-\frac{n}{2}} n^{-\frac{1}{2}\left(\frac{\nu}{\theta}-1\right)} n^{\frac{\nu}{2 \theta^{\prime}}}=2^{-\frac{n}{2}} n^{-\frac{\nu}{\theta}} n^{\frac{\nu+1}{2}} .
$$

Сопоставив $(2.14)$ с (2.10) при $\alpha=\frac{q}{2}\left(1+\frac{(\nu-1) \log n}{n}\right)$, видим, что $I_{2}<I_{1}$, и в силу (2.3) приходим к искомой оценке сверху в случае $r_{1}=\frac{1}{p}$.

Пусть теперь $r_{1}>\frac{1}{p}$. Здесь нам понадобится рассмотреть отдельно случаи $\theta \geqslant 2$ и $1 \leqslant \theta<2$.

Итак, пусть $\theta \geqslant 2$. Тогда полагаем

$$
\begin{aligned}
& \alpha=\left(r_{1}-\frac{1}{p}+\frac{1}{2}\right) /\left(r_{1}-\frac{1}{p}+\frac{1}{q}\right), \\
& N_{s}=\left[2^{n\left(r_{1}-\frac{1}{p}+1\right)} 2^{-(s, \gamma)\left(r_{1}-\frac{1}{p}\right)}\right]+1 .
\end{aligned}
$$

Отсюда

$$
\begin{aligned}
\sum_{n \leqslant\left(s, \gamma^{\prime}\right)<\alpha n} N_{s} & \ll n^{d}+2^{n\left(r_{1}-\frac{1}{p}+1\right)} \sum_{n \leqslant\left(s, \gamma^{\prime}\right)<\alpha n} 2^{-(s, \gamma)\left(r_{1}-\frac{1}{p}\right)} \\
& \ll n^{d}+2^{n\left(r_{1}-\frac{1}{p}+1\right)} 2^{-n\left(r_{1}-\frac{1}{p}\right)} n^{\nu-1}=n^{d}+2^{n} n^{\nu-1} \\
& \ll 2^{n} n^{\nu-1} \asymp M .
\end{aligned}
$$

Подставив в $(2.11)$ вместо $N_{s}$ их значения из $(2.16)$ и воспользовавшись затем неравенством Гёльдера с показателем $\theta / 2$ (с соответствующей модификацией при 
$\theta=2)$, получим

$$
\begin{aligned}
I_{1} \leqslant & 2^{-\frac{n}{2}\left(r_{1}-\frac{1}{p}+1\right)}\left(\sum_{n \leqslant\left(s, \gamma^{\prime}\right)<\alpha n} 2^{\frac{2}{p}(s, \gamma)} 2^{\left(r_{1}-\frac{1}{p}\right)(s, \gamma)}\left\|A_{s}(f, x)\right\|_{p}^{2}\right)^{\frac{1}{2}} \\
= & 2^{-\frac{n}{2}\left(r_{1}-\frac{1}{p}+1\right)}\left(\sum_{n \leqslant\left(s, \gamma^{\prime}\right)<\alpha n} 2^{2(s, r)}\left\|A_{s}(f, x)\right\|_{p}^{2} 2^{-(s, \gamma)\left(r_{1}-\frac{1}{p}\right)}\right)^{\frac{1}{2}} \\
\leqslant & 2^{-\frac{n}{2}\left(r_{1}-\frac{1}{p}+1\right)}\left(\sum_{n \leqslant\left(s, \gamma^{\prime}\right)<\alpha n} 2^{(s, r) \theta}\left\|A_{s}(f, x)\right\|_{p}^{\theta}\right)^{\frac{1}{\theta}} \\
& \times\left(\sum_{n \leqslant\left(s, \gamma^{\prime}\right)<\alpha n} 2^{-(s, \gamma)\left(r_{1}-\frac{1}{p}\right) \frac{\theta}{\theta-2}}\right)^{\frac{1}{2}-\frac{1}{\theta}} \ll 2^{-\frac{n}{2}\left(r_{1}-\frac{1}{p}+1\right)}\|f\|_{B_{p, \theta}^{r}} \\
& \times 2^{-\frac{n}{2}\left(r_{1}-\frac{1}{p}\right)} n^{(\nu-1)\left(\frac{1}{2}-\frac{1}{\theta}\right)} \leqslant 2^{-n\left(r_{1}-\frac{1}{p}+\frac{1}{2}\right)} n^{(\nu-1)\left(\frac{1}{2}-\frac{1}{\theta}\right)} .
\end{aligned}
$$

Если же $\theta=\infty$, то согласно теореме В будем иметь

$$
\begin{aligned}
I_{1} & \ll 2^{-\frac{n}{2}\left(r_{1}-\frac{1}{p}+1\right)}\left(\sum_{n \leqslant\left(s, \gamma^{\prime}\right)<\alpha n} 2^{\frac{2}{p}(s, \gamma)} 2^{\left(r_{1}-\frac{1}{p}\right)(s, \gamma)}\left\|A_{s}(f, x)\right\|_{p}^{2}\right)^{\frac{1}{2}} \\
& \ll 2^{-\frac{n}{2}\left(r_{1}-\frac{1}{p}+1\right)}\left(\sum_{n \leqslant\left(s, \gamma^{\prime}\right)<\alpha n} 2^{-\left(r_{1}-\frac{1}{p}\right)(s, \gamma)}\right)^{\frac{1}{2}} \\
& \ll 2^{-\frac{n}{2}\left(r_{1}-\frac{1}{p}+1\right)} 2^{-\frac{n}{2}\left(r_{1}-\frac{1}{p}\right)} n^{\frac{\nu-1}{2}}=2^{-n\left(r_{1}-\frac{1}{p}+\frac{1}{2}\right)} n^{\frac{\nu-1}{2}} .
\end{aligned}
$$

Сопоставив оценки (2.17) и (2.18) с оценкой $I_{2}$ (см. (2.10)) при $\alpha$, определенном в $(2.15)$; видим, что $I_{2}<I_{1}$, и, следовательно, согласно (2.3) будем иметь

$$
\begin{aligned}
\left\|f(x)-P\left(\Omega_{M}, x\right)\right\|_{q} & \ll 2^{-n\left(r_{1}-\frac{1}{p}+\frac{1}{2}\right)} n^{(\nu-1)\left(\frac{1}{2}-\frac{1}{\theta}\right)} \\
& \asymp\left(M^{-1} \log ^{\nu-1} M\right)^{r_{1}-\frac{1}{p}+\frac{1}{2}}\left(\log ^{\nu-1} M\right)^{\frac{1}{2}-\frac{1}{\theta}} .
\end{aligned}
$$

Если же $1 \leqslant \theta<2$, то полагаем

$$
\alpha=\left(\left(r_{1}-\frac{1}{p}+\frac{1}{2}\right)-(\nu-1)\left(\frac{1}{2}-\frac{1}{\theta}\right) \frac{\log n}{n}\right) /\left(r_{1}-\frac{1}{p}+\frac{1}{q}\right)
$$

и

$$
N_{s}=\left[2^{n\left(r_{1}-\frac{1}{p}+1\right)} n^{(\nu-1)} 2^{-(s, \gamma)\left(r_{1}-\frac{1}{p}\right)} 2^{(s, r) \theta}\left\|A_{s}(f, x)\right\|_{p}^{\theta}\right]+1 .
$$

В таком случае

$$
\begin{gathered}
\sum_{n \leqslant\left(s, \gamma^{\prime}\right)<\alpha n} N_{s} \ll n^{d}+2^{n\left(r_{1}-\frac{1}{p}+1\right)} n^{\nu-1} 2^{-n\left(r_{1}-\frac{1}{p}\right)} \sum_{n \leqslant\left(s, \gamma^{\prime}\right)<\alpha n} 2^{(s, r) \theta}\left\|A_{s}(f, x)\right\|_{p}^{\theta} \\
\leqslant n^{d}+2^{n} n^{\nu-1}\|f\|_{B_{p, \theta}^{r}}^{\theta} \ll 2^{n} n^{\nu-1} \asymp M .
\end{gathered}
$$


Подставив в соотношение (2.11) эти значения $N_{s}$, получим

$$
\begin{aligned}
I_{1} \leqslant & 2^{-\frac{n}{2}\left(r_{1}-\frac{1}{p}+1\right)} n^{-\frac{\nu-1}{2}}\left(\sum_{n \leqslant\left(s, \gamma^{\prime}\right)<\alpha n} 2^{\left(r_{1}+\frac{1}{p}\right)(s, \gamma)} 2^{-(s, r) \theta}\left\|A_{s}(f, x)\right\|_{p}^{2-\theta}\right)^{\frac{1}{2}} \\
\leqslant & 2^{-\frac{n}{2}\left(r_{1}-\frac{1}{p}+1\right)} n^{-\frac{\nu-1}{2}}\left(\sum_{n \leqslant\left(s, \gamma^{\prime}\right)<\alpha n} 2^{(s, r) \theta}\left\|A_{s}(f, x)\right\|_{p}^{\theta}\right)^{\frac{1}{\theta}-\frac{1}{2}} \\
& \times\left(\sum_{n \leqslant\left(s, \gamma^{\prime}\right)<\alpha n} 2^{-\left(r_{1}-\frac{1}{p}\right)(s, \gamma) \frac{\theta}{2 \theta-2}}\right)^{1-\frac{1}{\theta}} \\
& \ll 2^{-\frac{n}{2}\left(r_{1}-\frac{1}{p}+1\right)} n^{-\frac{\nu-1}{2}}\|f\|_{B_{p, \theta}^{r}}^{\frac{2-\theta}{2}} 2^{-\frac{n}{2}\left(r_{1}-\frac{1}{p}\right)} n^{(\nu-1)\left(1-\frac{1}{\theta}\right)} \\
\leqslant & 2^{-n\left(r_{1}-\frac{1}{p}+\frac{1}{2}\right)} n^{(\nu-1)\left(\frac{1}{2}-\frac{1}{\theta}\right)} .
\end{aligned}
$$

Легко видеть, что и в этом случае $I_{2} \leqslant I_{1}$ при значении $\alpha$, определенном в $(2.20)$, и поэтому согласно (2.3)

$$
\begin{aligned}
\left\|f(x)-P\left(\Omega_{M}, x\right)\right\|_{q} & \ll 2^{-n\left(r_{1}-\frac{1}{p}+\frac{1}{2}\right)} n^{(\nu-1)\left(\frac{1}{2}-\frac{1}{\theta}\right)} \\
& \asymp\left(M^{-1} \log ^{\nu-1} M\right)^{r_{1}-\frac{1}{p}+\frac{1}{2}}\left(\log ^{\nu-1} M\right)^{\frac{1}{2}-\frac{1}{\theta}} .
\end{aligned}
$$

Теперь перейдем к получению оценки сверху величины $e_{M}\left(B_{p, \theta}^{r}\right)_{q}$ в случае $\frac{1}{p}-$ $\frac{1}{q}<r_{1}<\frac{1}{p}$. Пусть сначала $\theta>q$. Тогда, положив

$$
\alpha=\frac{q}{2}-\left(\frac{q}{2}-1\right)(\nu-1) \frac{\log n}{n},
$$

будем иметь

$$
\begin{aligned}
I_{2} & \ll 2^{-\frac{q n}{2}\left(r_{1}-\frac{1}{p}+\frac{1}{q}\right)} n^{\left(\frac{q}{2}-1\right)(\nu-1)\left(r_{1}-\frac{1}{p}+\frac{1}{q}\right)} n^{(\nu-1)\left(\frac{1}{q}-\frac{1}{\theta}\right)} \\
& \asymp M^{-\frac{q}{2}\left(r_{1}-\frac{1}{p}+\frac{1}{q}\right)}\left(\log ^{\nu-1} M\right)^{(q-1)\left(r_{1}-\frac{1}{p}+\frac{1}{q}\right)}\left(\log ^{\nu-1} M\right)^{\frac{1}{q}-\frac{1}{\theta}} \\
& =M^{-\frac{q}{2}\left(r_{1}-\frac{1}{p}+\frac{1}{q}\right)}\left(\log ^{\nu-1} M\right)^{(q-1)\left(r_{1}-\frac{1}{p}+\frac{q^{\prime}}{q \theta^{\prime}}\right)} .
\end{aligned}
$$

Оценим $I_{1}$. С этой целью положим

$$
N_{s}=\left[2^{n} 2^{(s, \tilde{\gamma})\left(\frac{1}{p}-r_{1}\right)} 2^{-\alpha n\left(\frac{1}{p}-r_{1}\right)}\right]+1,
$$

где координаты вектора $\tilde{\gamma}=\left(\tilde{\gamma}_{1}, \ldots, \tilde{\gamma}_{d}\right)$ удовлетворяют соотношениям

$$
\gamma_{1}^{\prime}=\tilde{\gamma}_{1}=\cdots=\gamma_{\nu}^{\prime}=\tilde{\gamma}_{\nu}, \quad 1<\tilde{\gamma}_{j}<\gamma_{j}^{\prime}, \quad j=\overline{\nu+1, d} .
$$

Воспользовавшись леммой В, получим

$$
\begin{aligned}
\sum_{n \leqslant\left(s, \gamma^{\prime}\right)<\alpha n} N_{s} & \leqslant 2^{-\alpha n\left(\frac{1}{p}-r_{1}\right)+n} \sum_{n \leqslant\left(s, \gamma^{\prime}\right)<\alpha n} 2^{(s, \tilde{\gamma})\left(\frac{1}{p}-r_{1}\right)}+n^{d} \\
& \ll 2^{-\alpha n\left(\frac{1}{p}-r_{1}\right)+n} 2^{\alpha n\left(\frac{1}{p}-r_{1}\right)} n^{\nu-1}+n^{d} \ll 2^{n} n^{\nu-1} \asymp M .
\end{aligned}
$$


Таким образом, подставив в (2.11) $N_{s}$, определенные соотношением $(2.23)$, и $\alpha$, определенное равенством (2.21), будем иметь при $q<\theta<\infty$

$$
\begin{aligned}
& I_{1} \ll 2^{\frac{\alpha n}{2}\left(\frac{1}{p}-r_{1}\right)-\frac{n}{2}}\left(\sum_{n \leqslant\left(s, \gamma^{\prime}\right)<\alpha n} 2^{(s, \tilde{\gamma})\left(\frac{1}{p}+r_{1}\right)}\left\|A_{s}(f, x)\right\|_{p}^{2}\right)^{\frac{1}{2}} \\
& \ll 2^{-\frac{n}{2}+\frac{\alpha n}{2}\left(\frac{1}{p}-r_{1}\right)}\left(\sum_{n \leqslant\left(s, \gamma^{\prime}\right)<\alpha n} 2^{2(s, r)}\left\|A_{s}(f, x)\right\|_{p}^{2} 2^{-(s, \tilde{\gamma})\left(-\frac{1}{p}+r_{1}\right)}\right)^{\frac{1}{2}} \\
& \leqslant 2^{-\frac{n}{2}+\frac{\alpha n}{2}\left(\frac{1}{p}-r_{1}\right)}\left(\sum_{n \leqslant\left(s, \gamma^{\prime}\right)<\alpha n} 2^{(s, r) \theta}\left\|A_{s}(f, x)\right\|_{p}^{\theta}\right)^{\frac{1}{\theta}} \\
& \times\left(\sum_{n \leqslant\left(s, \gamma^{\prime}\right)<\alpha n} 2^{-(s, \tilde{\gamma})\left(-\frac{1}{p}+r_{1}\right) \frac{\theta}{\theta-2}}\right)^{\frac{1}{2}-\frac{1}{\theta}} \\
& \leqslant 2^{-\frac{n}{2}+\frac{\alpha n}{2}\left(\frac{1}{p}-r_{1}\right)}\|f\|_{B_{p, \theta}^{r}}\left(\sum_{n \leqslant\left(s, \gamma^{\prime}\right)<\alpha n} 2^{(s, \tilde{\gamma})\left(\frac{1}{p}-r_{1}\right) \frac{\theta}{\theta-2}}\right)^{\frac{1}{2}-\frac{1}{\theta}} \\
& \ll 2^{-\frac{n}{2}+\frac{\alpha n}{2}\left(\frac{1}{p}-r_{1}\right)} 2^{\frac{\alpha n}{2}\left(\frac{1}{p}-r_{1}\right)} n^{(\nu-1)\left(\frac{1}{2}-\frac{1}{\theta}\right)} \\
& =2^{-\frac{n}{2}+\alpha n\left(\frac{1}{p}-r_{1}\right)} n^{(\nu-1)\left(\frac{1}{2}-\frac{1}{\theta}\right)} \\
& \leqslant 2^{-\frac{n}{2}+\frac{q n}{2}\left(\frac{1}{p}-r_{1}\right)} n^{-(\nu-1)\left(\frac{q}{2}-1\right)\left(\frac{1}{p}-r_{1}\right)} n^{(\nu-1)\left(\frac{1}{2}-\frac{1}{\theta}\right)} \\
& \asymp M^{-\frac{q}{2}\left(r_{1}-\frac{1}{p}+\frac{1}{q}\right)}\left(\log ^{\nu-1} M\right)^{(q-1)\left(r_{1}-\frac{1}{p}+\frac{q^{\prime}}{q \theta^{\prime}}\right)} .
\end{aligned}
$$

Если $\theta=\infty$, то согласно теореме В для $f \in B_{p, \infty}^{r}$ имеем $\left\|A_{s}(f, x)\right\|_{p} \ll 2^{-(s, r)}$ и тогда вследствие (2.11)

$$
\begin{aligned}
I_{1} & \ll 2^{\frac{\alpha n}{2}\left(\frac{1}{p}-r_{1}\right)-\frac{n}{2}}\left(\sum_{n \leqslant\left(s, \gamma^{\prime}\right)<\alpha n} 2^{(s, \tilde{\gamma})\left(r_{1}+\frac{1}{p}\right)} 2^{-(s, \tilde{\gamma}) 2 r_{1}}\right)^{\frac{1}{2}} \\
& =2^{-\frac{n}{2}+\frac{\alpha n}{2}\left(\frac{1}{p}-r_{1}\right)}\left(\sum_{n \leqslant\left(s, \gamma^{\prime}\right)<\alpha n} 2^{(s, \tilde{\gamma})\left(\frac{1}{p}-r_{1}\right)}\right)^{\frac{1}{2}} \\
& \ll 2^{-\frac{n}{2}+\frac{\alpha n}{2}\left(\frac{1}{p}-r_{1}\right)} 2^{\frac{\alpha n}{2}\left(\frac{1}{p}-r_{1}\right)} n^{\frac{\nu-1}{2}}=2^{-\frac{n}{2}+\alpha n\left(\frac{1}{p}-r_{1}\right)} n^{\frac{\nu-1}{2}} \\
& \asymp M^{-\frac{q}{2}\left(r_{1}-\frac{1}{p}+\frac{1}{q}\right)}\left(\log ^{\nu-1} M\right)^{(q-1)\left(r_{1}-\frac{1}{p}+\frac{q^{\prime}}{q}\right)} .
\end{aligned}
$$

Таким образом, подставив оценки $(2.24),(2.25)$ и $(2.22)$ в $(2.3)$, приходим к искомой оценке сверху $e_{M}\left(B_{p, \theta}^{r}\right)_{q}$ в случае $\theta>q$.

Для завершения оценок сверху осталось рассмотреть случай $1 \leqslant \theta<q$. По заданному $M$ подберем $n$ из соотношения $M \asymp 2^{n} n^{\nu-1}$ и определим числа

$$
\begin{aligned}
& n_{1}=\frac{q}{2} n-\left(\frac{q}{2}-1\right)(\nu-1) \log n \\
& n_{2}=\frac{q}{2} n+\frac{q}{2}(\nu-1) \log n
\end{aligned}
$$


Функцию $f \in B_{p, \theta}^{r}$ представим в виде

$$
f(x)=\left(\sum_{(s, \gamma)<n}+\sum_{n \leqslant(s, \gamma)<n_{1}}+\sum_{n_{1} \leqslant(s, \gamma)<n_{2}}+\sum_{(s, \gamma) \geqslant n_{2}}\right) \delta_{s}(f, x)
$$

и заметим, что согласно теореме Литтлвуда-Пэли каждое слагаемое в (2.26), с соответствуюшими постоянными, принадлежит классу $B_{p, \theta}^{r}$.

Приближаюший полином $P\left(\Omega_{M}, x\right)$ для $f \in B_{p, \theta}^{r}$ будем подбирать в виде

$$
P\left(\Omega_{M}, x\right)=\sum_{(s, \gamma)<n} \delta_{s}(f, x)+\sum_{n \leqslant(s, \gamma)<n_{1}} P_{1}\left(\Omega_{N_{s}}, x\right)+\sum_{n_{1} \leqslant(s, \gamma)<n_{2}} P_{2}\left(\Omega_{M_{s}}, x\right),
$$

где $P_{1}\left(\Omega_{N_{s}}, x\right)$ и $P_{2}\left(\Omega_{M_{s}}, x\right)$ - полиномы, которые будут сконструированы в процессе установления оценки.

Итак, предположим, что искомый полином $P\left(\Omega_{M}, x\right)$ построен. Тогда согласно $(2.26)$ и $(2.27)$ можем записать

$$
\begin{aligned}
\left\|f(x)-P\left(\Omega_{M}, x\right)\right\|_{q} \leqslant & \left\|\sum_{n \leqslant(s, \gamma)<n_{1}}\left(\delta_{s}(f, x)-P_{1}\left(\Omega_{N_{s}}, x\right)\right)\right\|_{q} \\
& +\left\|\sum_{n_{1} \leqslant(s, \gamma)<n_{2}}\left(\delta_{s}(f, x)-P_{2}\left(\Omega_{M_{s}}, x\right)\right)\right\|_{q} \\
& +\left\|\sum_{(s, \gamma) \geqslant n_{2}} \delta_{s}(f, x)\right\|_{q}=I_{4}+I_{5}+I_{6} .
\end{aligned}
$$

Проведем оценки каждого из полученных слагаемых.

Повторив те же рассуждения, что и при оценке $I_{1}$, получим

$$
I_{4} \ll\left(\sum_{n \leqslant(s, \gamma)<n_{1}} 2^{\frac{2}{p}\|s\|_{1}} N_{s}^{-1}\left\|A_{s}(f, x)\right\|_{p}^{2}\right)^{\frac{1}{2}} .
$$

Числа $N_{s}$ выберем следующим образом:

$$
N_{s}=\left[2^{n} n^{\nu-1} 2^{n_{1}\left(r_{1}-\frac{1}{p}\right)} 2^{\left(s, \gamma^{\prime}\right)\left(\frac{1}{p}-r_{1}\right)} 2^{(s, r)}\left\|A_{s}(f, x)\right\|_{p} n_{1}^{-\frac{\nu-1}{\theta^{\prime}}}\right]+1 .
$$

Тогда согласно неравенству Гёльдера (с естественной модификацией при $\theta=1$ ) и 
лемме В будем иметь

$$
\begin{aligned}
& \sum_{n \leqslant(s, \gamma)<n_{1}} N_{s} \ll n_{1}^{d}+2^{n} n^{\nu-1} 2^{n_{1}\left(r_{1}-\frac{1}{p}\right)} n_{1}^{-\frac{\nu-1}{\theta^{\prime}}} \\
& \times \sum_{n \leqslant(s, \gamma)<n_{1}} 2^{\left(s, \gamma^{\prime}\right)\left(\frac{1}{p}-r_{1}\right)} 2^{(s, r)}\left\|A_{s}(f, x)\right\|_{p} \\
& \leqslant n_{1}^{d}+2^{n} n^{\nu-1} 2^{n_{1}\left(r_{1}-\frac{1}{p}\right)} n_{1}^{-\frac{\nu-1}{\theta^{\prime}}}\left(\sum_{n \leqslant\left(s, \gamma^{\prime}\right)<n_{1}} 2^{(s, r) \theta}\left\|A_{s}(f, x)\right\|_{p}^{\theta}\right)^{\frac{1}{\theta}} \\
& \times\left(\sum_{n \leqslant(s, \gamma)<n_{1}} 2^{\left(s, \gamma^{\prime}\right)\left(\frac{1}{p}-r_{1}\right) \theta^{\prime}}\right)^{\frac{1}{\theta^{\prime}}} \\
& \ll n_{1}^{d}+2^{n} n^{\nu-1} 2^{n_{1}\left(r_{1}-\frac{1}{p}\right)} n_{1}^{-\frac{\nu-1}{\theta^{\prime}}}\|f\|_{B_{p, \theta}^{r}} 2^{n_{1}\left(\frac{1}{p}-r_{1}\right)} n_{1}^{\frac{\nu-1}{\theta^{\prime}}} \\
& \ll 2^{n} n^{\nu-1} \asymp M \text {. }
\end{aligned}
$$

Теперь, подставив в (2.29) эти значения $N_{s}$ и выполнив соответствующие преобразования, получим

$$
\begin{aligned}
I_{4} & \ll 2^{-\frac{n}{2}} n_{1}^{-\frac{\nu-1}{2}} 2^{\frac{n_{1}}{2}\left(\frac{1}{p}-r_{1}\right)} n_{1}^{\frac{\nu-1}{2 \theta^{\prime}}}\left(\sum_{n \leqslant(s, \gamma)<n_{1}} 2^{(s, r)}\left\|A_{s}(f, x)\right\|_{p} 2^{\left(s, \gamma^{\prime}\right)\left(\frac{1}{p}-r_{1}\right)}\right)^{\frac{1}{2}} \\
& \ll 2^{\frac{n_{1}}{2}\left(\frac{1}{p}-r_{1}\right)-\frac{n}{2}} n_{1}^{\frac{\nu-1}{2}\left(\frac{1}{\theta^{\prime}}-1\right)} \asymp M^{-\frac{q}{2}\left(r_{1}-\frac{1}{p}+\frac{1}{q}\right)}\left(\log ^{\nu-1} M\right)^{(q-1)\left(r_{1}-\frac{1}{p}+\frac{q^{\prime}}{q \theta^{\prime}}\right)} .
\end{aligned}
$$

Переходя к оценке $I_{5}$, проделаем следуюшую процедуру. Каждому $l \in \mathbb{N}, n_{1} \leqslant$ $l<n_{2}$, поставим в соответствие числа

$$
\begin{gathered}
S_{l}=\left(\sum_{l \leqslant(s, \gamma)<l+1} 2^{(s, r) \theta}\left\|A_{s}(f, x)\right\|_{p}^{\theta}\right)^{\frac{1}{\theta}}, \\
m_{l}=\left[2^{-l \frac{q^{\prime}}{q}} S_{l}^{\theta} 2^{n \frac{q^{\prime}}{2}} n^{(\nu-1) \frac{q^{\prime}}{2}}\right] .
\end{gathered}
$$

Пусть

$$
\sum_{n_{1} \leqslant(s, \gamma)<n_{2}} \delta_{s}(f, x)=\sum_{n_{1} \leqslant l<n_{2}}\left(\sum_{l \leqslant(s, \gamma)<l+1}^{\prime} \delta_{s}(f, x)+\sum_{l \leqslant(s, \gamma)<l+1}^{\prime \prime} \delta_{s}(f, x)\right),
$$

где первая сумма внутри скобок содержит $m_{l}$ блоков $\delta_{s}(f, x)$ по тем $s=\left(s_{1}, \ldots, s_{d}\right)$, которым соответствуют наибольшие значения $\left\|A_{s}(f, x)\right\|_{p}$, а вторая - остальные. Приближающие полиномы $P_{2}\left(\Omega_{M_{s}}, x\right)$ будем строить согласно лемме 2.1 только для тех блоков $\delta_{s}(f, x)$, которые попали в первую сумму. 
Таким образом, будем иметь

$$
\begin{aligned}
I_{5} & \ll\left(\sum_{l=n_{1}}^{n_{2}} \sum_{l \leqslant(s, \gamma)<l+1}^{\prime} 2^{\|s\|_{1}} M_{s}^{-1}\left\|\delta_{s}(f, x)\right\|_{2}^{2}\right)^{\frac{1}{2}}+\left\|\sum_{l=n_{1}}^{n_{2}} \sum_{l \leqslant(s, \gamma)<l+1}^{\prime \prime} \delta_{s}(f, x)\right\|_{q} \\
& \ll\left(\sum_{l=n_{1}}^{n_{2}} 2^{l} \sum_{l \leqslant(s, \gamma)<l+1}^{\prime} M_{s}^{-1}\left\|A_{s}(f, x)\right\|_{2}^{2}\right)^{\frac{1}{2}}+\left\|\sum_{l=n_{1}}^{n_{2}} \sum_{l \leqslant(s, \gamma)<l+1}^{\prime \prime} \delta_{s}(f, x)\right\|_{q} \\
& =\mathscr{J}_{1}+\mathscr{J}_{2} .
\end{aligned}
$$

Оценим слагаемое $\mathscr{J}_{1}$, предположив сначала, что $r_{1} \geqslant \frac{1}{p}-\frac{q^{\prime}}{q \theta^{\prime}}$. Для каждого вектора $s=\left(s_{1}, \ldots, s_{d}\right), l \leqslant(s, \gamma)<l+1, n_{1} \leqslant l<n_{2}$, положим

$$
M_{s}=\left[2^{\frac{l}{2}}\left\|A_{s}(f, x)\right\|_{2} 2^{n_{1}\left(r_{1}-\frac{1}{p}+\frac{q^{\prime}}{q \theta^{\prime}}\right)}\left(2^{n} n^{\nu-1}\right)^{1-\frac{q^{\prime}}{2 \theta^{\prime}}}\right]+1 .
$$

Тогда

$$
\begin{aligned}
\sum_{l=n_{1}}^{n_{2}} \sum_{l \leqslant(s, \gamma)<l+1}^{\prime} M_{s} \ll & n_{2}^{d}+2^{n_{1}\left(r_{1}-\frac{1}{p}+\frac{q^{\prime}}{q \theta^{\prime}}\right)}\left(2^{n} n^{\nu-1}\right)^{1-\frac{q^{\prime}}{2 \theta^{\prime}}} \\
& \times \sum_{l=n_{1}}^{n_{2}} 2^{\frac{l}{2}} \sum_{l \leqslant(s, \gamma)<l+1}^{\prime}\left\|A_{s}(f, x)\right\|_{2} .
\end{aligned}
$$

Далее, принимая во внимание, что во внутренней сумме правой части (2.36) содержится $m_{l}$ слагаемых, и воспользовавшись неравенством (1.5) и неравенством Гёльдера (с естественной модификацией при $\theta=1$ ), получим

$$
\begin{aligned}
\sum_{l=n_{1}}^{n_{2}} 2^{\frac{l}{2}} & \sum_{l \leqslant(s, \gamma)<l+1}^{\prime}\left\|A_{s}(f, x)\right\|_{2} \\
& \ll \sum_{l=n_{1}}^{n_{2}} 2^{-l\left(r_{1}-\frac{1}{p}\right)}\left(\sum_{l \leqslant(s, \gamma)<l+1}^{\prime} 2^{(s, r) \theta}\left\|A_{s}(f, x)\right\|_{p}^{\theta}\right)^{\frac{1}{\theta}} m_{l}^{\frac{1}{\theta^{\prime}}} \\
& \leqslant \sum_{l=n_{1}}^{n_{2}} 2^{-l\left(r_{1}-\frac{1}{p}\right)} S_{l} m_{l}^{\frac{1}{\theta^{\prime}}} \leqslant 2^{\frac{n q^{\prime}}{2 \theta^{\prime}}} n^{(\nu-1) \frac{q^{\prime}}{2 \theta^{\prime}}} \sum_{l=n_{1}}^{n_{2}} 2^{-l\left(r_{1}-\frac{1}{p}+\frac{q}{q \theta^{\prime}}\right)} S_{l}^{\theta} \\
& \leqslant 2^{\frac{n q^{\prime}}{2 \theta^{\prime}}} n^{(\nu-1) \frac{q^{\prime}}{2 \theta^{\prime}}} 2^{-n_{1}\left(r_{1}-\frac{1}{p}+\frac{q^{\prime}}{q \theta^{\prime}}\right)} \sum_{l=n_{1}}^{n_{2}} S_{l}^{\theta} \\
& \leqslant 2^{-\frac{n q}{2}\left(r_{1}-\frac{1}{p}\right)} n^{(\nu-1) \frac{q^{\prime}}{2 \theta^{\prime}}} n^{(\nu-1)\left(\frac{q}{2}-1\right)\left(r_{1}-\frac{1}{p}+\frac{q^{\prime}}{q \theta^{\prime}}\right)}
\end{aligned}
$$

Подставив (2.37) в (2.36) и выполнив несложные преобразования, приходим к оценке

$$
\sum_{l=n_{1} l \leqslant(s, \gamma)<l+1}^{n_{2}} \sum_{s}^{\prime} M \ll 2^{n} n^{\nu-1} \asymp M .
$$


Таким образом, подставив $M_{s}$, определенные в $(2.35)$, и воспользовавшись оценкой (2.37), получим

$$
\begin{aligned}
\mathscr{J}_{1} & \ll\left(\sum_{l=n_{1}}^{n_{2}} 2^{\frac{l}{2}} \sum_{l \leqslant(s, \gamma)<l+1}\left\|A_{s}(f, x)\right\|_{2}\right)^{\frac{1}{2}} 2^{-\frac{n_{1}}{2}\left(r_{1}-\frac{1}{p}+\frac{q^{\prime}}{q \theta^{\prime}}\right)}\left(2^{n} n^{\nu-1}\right)^{\frac{q^{\prime}}{4 \theta^{\prime}}-\frac{1}{2}} \\
& \ll 2^{-\frac{n q}{2}\left(r_{1}-\frac{1}{p}+\frac{1}{q}\right)} n^{(\nu-1)\left(\frac{q}{2}-1\right)\left(r_{1}-\frac{1}{p}+\frac{q^{\prime}}{q \theta^{\prime}}\right)} n^{(\nu-1)\left(\frac{q^{\prime}}{\theta^{\prime}}-1\right)} \\
& \asymp M^{-\frac{q}{2}\left(r_{1}-\frac{1}{p}+\frac{1}{q}\right)}\left(\log ^{\nu-1} M\right)^{(q-1)\left(r_{1}-\frac{1}{p}+\frac{q^{\prime}}{q \theta^{\prime}}\right)} .
\end{aligned}
$$

Если же имеет место случай $\frac{1}{p}-\frac{1}{q}<r_{1}<\frac{1}{p}-\frac{q^{\prime}}{q \theta^{\prime}}$, то каждому вектору $s=$ $\left(s_{1}, \ldots, s_{d}\right), l \leqslant(s, \gamma)<l+1, n_{1} \leqslant l<n_{2}$, поставим в соответствие число

$$
M_{s}=\left[2^{\frac{l}{2}}\left\|A_{s}(f, x)\right\|_{2} 2^{n_{2}\left(r_{1}-\frac{1}{p}+\frac{q^{\prime}}{q \theta^{\prime}}\right)}\left(2^{n} n^{\nu-1}\right)^{1-\frac{q^{\prime}}{2 \theta^{\prime}}}\right]+1 .
$$

С помошью рассуждений, приводимых при выводе (2.36) и (2.37), убеждаемся в том, что и в данном случае справедливо соотношение

$$
\sum_{l=n_{1}}^{n_{2}} \sum_{l \leqslant(s, \gamma)<l+1}^{\prime} M_{s} \ll M .
$$

Подставив в выражение для $\mathscr{J}_{1}$ значения $M_{s}$ из $(2.39)$ и выполнив ряд преобразований, аналогичных тем, что и в случае $r_{1} \geqslant \frac{1}{p}-\frac{q^{\prime}}{q \theta^{\prime}}$, получим

$$
\mathscr{J}_{1} \ll M^{-\frac{q}{2}\left(r_{1}-\frac{1}{p}+\frac{1}{q}\right)}\left(\log ^{\nu-1} M\right)^{\frac{1}{2}\left(\frac{q^{\prime}}{\theta^{\prime}}-1\right)} \leqslant M^{-\frac{q}{2}\left(r_{1}-\frac{1}{p}+\frac{1}{q}\right)} .
$$

Таким образом, объединив $(2.38)$ с (2.40), находим

$$
\mathscr{J}_{1} \ll M^{-\frac{q}{2}\left(r_{1}-\frac{1}{p}+\frac{1}{q}\right)}\left(\log ^{\nu-1} M\right)^{(q-1)\left(r_{1}-\frac{1}{p}+\frac{q^{\prime}}{q \theta^{\prime}}\right)_{+}} .
$$

Оценим $\mathscr{J}_{2}$. Здесь мы будем пользоваться леммой А. Занумеруем "блоки" $\delta_{s}(f, x)$, входящие в $\mathscr{J}_{2}$, в порядке убьвания норм $\left\|A_{s}(f, x)\right\|_{p}$, обозначив их $a_{i}(f, l)$, $i=1,2, \ldots$. Тогда, принимая во внимание определение $S_{l}$ (см. (2.31)), можем записать

$$
a_{i}(f, l) \leqslant i^{-\frac{1}{\theta}} 2^{-l r_{1}} S_{l}
$$

и, следовательно, в силу леммы А

$$
\begin{aligned}
\mathscr{J}_{2} & \ll\left(\sum_{l=n_{1}}^{n_{2}} \sum_{i>m_{l}} a_{i}^{q}(f, l) 2^{l\left(\frac{1}{p}-\frac{1}{q}\right) q}\right)^{\frac{1}{q}} \\
& =\left(\sum_{l=n_{1}}^{n_{2}} \sum_{i>m_{l}} a_{i}^{\theta}(f, l) a_{i}^{q-\theta}(f, l) 2^{l\left(\frac{1}{p}-\frac{1}{q}\right) q}\right)^{\frac{1}{q}} \\
& \ll\left(\sum_{l=n_{1}}^{n_{2}} m_{l}^{-\frac{q-\theta}{\theta}} 2^{-l(q-\theta) r_{1}} S_{l}^{q-\theta} 2^{l\left(\frac{1}{p}-\frac{1}{q}\right) q} \sum_{l \leqslant(s, \gamma)<l+1} 2^{(s, r) \theta}\left\|A_{s}(f, x)\right\|_{p}^{\theta}\right)^{\frac{1}{q}} \\
& \ll\left(\sum_{l=n_{1}}^{n_{2}} m_{l}^{-\frac{q-\theta}{\theta}} 2^{-l\left(r_{1}-\frac{1}{p}+\frac{1}{q}\right)} S_{l}^{q}\right)^{\frac{1}{q}} .
\end{aligned}
$$


Теперь, подставив вместо $m_{l}$ их значения из $(2.32)$, получим

$$
\mathscr{J}_{2} \ll\left(2^{-\frac{n q^{\prime}}{2} \cdot \frac{q-\theta}{\theta}} n^{-(\nu-1) \frac{q^{\prime}}{2} \cdot \frac{q-\theta}{\theta}}\right)^{\frac{1}{q}}\left(\sum_{l=n_{1}}^{n_{2}} 2^{-l q\left(r_{1}-\frac{1}{p}+\frac{q^{\prime}}{q \theta^{\prime}}\right)} S_{l}^{\theta}\right)^{\frac{1}{q}} .
$$

Отсюда после несложных преобразований находим

$$
\mathscr{J}_{2} \ll M^{-\frac{q}{2}\left(r_{1}-\frac{1}{p}+\frac{1}{q}\right)}\left(\log ^{\nu-1} M\right)^{(q-1)\left(r_{1}-\frac{1}{p}+\frac{q^{\prime}}{q \theta^{\prime}}\right)}
$$

в случае $r_{1} \geqslant \frac{1}{p}-\frac{q^{\prime}}{q \theta^{\prime}}$ и

$$
\mathscr{J}_{2} \ll M^{-\frac{q}{2}\left(r_{1}-\frac{1}{p}+\frac{1}{q}\right)}
$$

в случае $\frac{1}{p}-\frac{1}{q}<r_{1}<\frac{1}{p}-\frac{q^{\prime}}{q \theta^{\prime}}$. Подставив полученные оценки $(2.41)-(2.43)$ в $(2.34)$, находим

$$
I_{5} \ll M^{-\frac{q}{2}\left(r_{1}-\frac{1}{p}+\frac{1}{q}\right)}\left(\log ^{\nu-1} M\right)^{(q-1)\left(r_{1}-\frac{1}{p}+\frac{q^{\prime}}{q \theta^{\prime}}\right)_{+}} .
$$

Наконец, для оценки сверху $I_{6}$, повторив рассуждения, которые использовались при нахождении оценки $I_{2}$, будем иметь

$$
I_{6} \ll 2^{-n_{2}\left(r_{1}-\frac{1}{p}+\frac{1}{q}\right)} n^{(\nu-1)\left(\frac{1}{q}-\frac{1}{\theta}\right)} \asymp M^{-\frac{q}{2}\left(r_{1}-\frac{1}{p}+\frac{1}{q}\right)}\left(\log ^{\nu-1} M\right)^{(q-1)\left(r_{1}-\frac{1}{p}+\frac{q^{\prime}}{q \theta^{\prime}}\right)} .
$$

Следовательно, подставив (2.45), (2.44) и (2.30) в (2.28), приходим к требуемой оценке сверху $e_{M}\left(B_{p, \theta}^{r}\right)_{q}$ и в случае $\frac{1}{p}-\frac{1}{q}<r_{1}<\frac{1}{p}, 1 \leqslant \theta<q$.

Таким образом, все оценки сверху в теореме доказаны.

При нахождении соответствуюших оценок снизу будем пользоваться двойственным соотношением, которое вытекает из более общего результата С. М. Никольского (см., например, [34, с. 25]).

Пусть $f \in L_{q}\left(\pi_{d}\right)$; тогда

$$
e_{M}(f)_{q}=\inf _{\Omega_{M}} \sup _{\substack{P \in L^{\perp}\left(\Omega_{M}\right) \\\|P\|_{q^{\prime}} \leqslant 1}}\left|\int_{\pi_{d}} f(x) P(x) d x\right|
$$

где $L^{\perp}\left(\Omega_{M}\right)$ - множество функций, ортогональных подпространству тригонометрических полиномов с "номерами" гармоник из множества $\Omega_{M}$. Отметим также, что оценки снизу достаточно установить при $\nu=d$.

Итак, рассмотрим последовательно все случаи теоремы.

Пусть $r_{1}=\frac{1}{p}$. Тогда по заданному $M$ подберем $l$ из соотношения

$$
l=\frac{q}{2} \log M-(q-1)(d-1) \log \log M
$$

и рассмотрим функцию

$$
g_{1}(x)=\sum_{(s, 1) \leqslant l} \prod_{j=1}^{d} \sum_{k_{j} \in \rho^{+}\left(s_{j}\right)} k_{j}^{-1} \cos k_{j} x_{j}
$$


где $\rho^{+}\left(s_{j}\right)=\left\{k_{j}: 2^{s_{j}-1} \leqslant k_{j}<2^{s_{j}}\right\}$.

Оценим сначала $\left\|g_{1}\right\|_{B_{p, \theta}^{r}}$ при $1<p<2$. С этой целью введем обозначения

$$
\begin{aligned}
\delta_{s}^{+}\left(g_{1}, x\right) & =\prod_{j=1}^{d} \sum_{k_{j} \in \rho^{+}\left(s_{j}\right)} k_{j}^{-1} \cos k_{j} x_{j}, \\
d_{s}(x) & =\prod_{j=1}^{d} \sum_{k_{j} \in \rho^{+}\left(s_{j}\right)} \cos k_{j} x_{j} .
\end{aligned}
$$

Поскольку $\left\|d_{s}(x)\right\|_{p} \asymp 2^{(s, 1)\left(1-\frac{1}{p}\right)}$, то согласно неравенству Бернштейна (см., например, [3, с. 14])

$$
\left\|\delta_{s}^{+}\left(g_{1}, x\right)\right\|_{p} \ll 2^{-(s, 1)}\left\|d_{s}(x)\right\|_{p} \asymp 2^{-(s, 1) \frac{1}{p}} .
$$

Следовательно, принимая во внимание то, что $r_{1}=\frac{1}{p}$, будем иметь

$$
\begin{aligned}
\left\|g_{1}\right\|_{B_{p, \theta}^{r}} & =\left(\sum_{(s, 1) \leqslant l} 2^{(s, r) \theta}\left\|\delta_{s}^{+}\left(g_{1}, x\right)\right\|_{p}^{\theta}\right)^{\frac{1}{\theta}}=\left(\sum_{(s, 1) \leqslant l} 2^{(s, 1) \frac{\theta}{p}}\left\|\delta_{s}^{+}\left(g_{1}, x\right)\right\|_{p}^{\theta}\right)^{\frac{1}{\theta}} \\
& \ll\left(\sum_{(s, 1) \leqslant l} 1\right)^{\frac{1}{\theta}} \ll l^{\frac{d}{\theta}}
\end{aligned}
$$

при $1 \leqslant \theta<\infty$. Если же $\theta=\infty$, то согласно $(2.47)$

$$
\left\|g_{1}\right\|_{B_{p, \infty}^{r}}=\sup _{s} 2^{(s, r)}\left\|\delta_{s}^{+}(g, x)\right\|_{p} \ll \sup _{s} 2^{(s, 1) \frac{1}{p}} 2^{-(s, 1) \frac{1}{p}}=1 .
$$

Пусть теперь $p=1$. Тогда заметим, что $r=(1, \ldots, 1) \in \mathbb{N}^{d}$, и рассмотрим функцию

$$
F(x)=\sum_{k>0} \prod_{j=1}^{d} k_{j}^{-1} \cos k_{j} x_{j} .
$$

Известно (см., например, [3, с. 39]), что эта функция принадлежит классу $b H_{1}^{r}$, $b>0, r=(1, \ldots, 1) \in \mathbb{N}^{d}$, и поэтому согласно теореме $\mathrm{B}$

$$
\left\|A_{s}(F, x)\right\|_{1} \ll 2^{-(s, 1)} .
$$

Следовательно, при $r=(1, \ldots, 1) \in \mathbb{N}^{d}$ и $1 \leqslant \theta<\infty$ будем иметь

$$
\begin{aligned}
\left\|g_{1}\right\|_{B_{1, \theta}^{r}} & =\left(\sum_{(s, 1) \leqslant l} 2^{(s, 1) \theta}\left\|A_{s}\left(g_{1}, x\right)\right\|_{1}^{\theta}\right)^{\frac{1}{\theta}}=\left(\sum_{(s, 1) \leqslant l} 2^{(s, 1) \theta}\left\|A_{s}(F, x)\right\|_{1}^{\theta}\right)^{\frac{1}{\theta}} \\
& \ll\left(\sum_{(s, 1) \leqslant l} 1\right)^{\frac{1}{\theta}} \ll l^{\frac{d}{\theta}}
\end{aligned}
$$


Аналогично, если $\theta=\infty$, то

$\left\|g_{1}\right\|_{B_{1, \infty}^{r}}=\sup _{s} 2^{(s, r)}\left\|A_{s}\left(g_{1}, x\right)\right\|_{1}=\sup _{s} 2^{(s, 1)}\left\|A_{s}(F, x)\right\|_{1} \ll \sup _{s} 2^{(s, 1)} 2^{-(s, 1)}=1$.

Из $(2.48),\left(2.48^{\prime}\right),(2.49)$ и $\left(2.49^{\prime}\right)$ заключаем, что функция

$$
f_{1}(x)=C_{1} l^{-\frac{d}{\theta}} g_{1}(x), \quad C_{1}>0,
$$

принадлежит классу $B_{p, \theta}^{r}, r=\left(\frac{1}{p}, \ldots, \frac{1}{p}\right) \in \mathbb{R}_{+}^{d}, 1 \leqslant p<2$.

Теперь построим функцию $P_{1}(x)$, которая удовлетворяла бы требованиям, предъявляемым к $P(x)$ из $(2.46)$. Пусть

$$
v_{1}(x)=\sum_{(s, 1) \leqslant l} \prod_{j=1}^{d} \sum_{k_{j} \in \rho^{+}\left(s_{j}\right)} \cos k_{j} x_{j}
$$

и $\Omega_{M}$ - произвольный набор из $M$ векторов $k=\left(k_{1}, \ldots, k_{d}\right)$ с целочисленными координатами. Обозначим через

$$
u_{1}(x)=\sum_{k \in \Omega_{M}}^{*} \prod_{j=1}^{d} \cos k_{j} x_{j}
$$

функцию, содержащую только те слагаемые из (2.50), которые имеют "номера" из множества $\Omega_{M}$, и положим $w_{1}(x)=v_{1}(x)-u_{1}(x)$. В таком случае при $1<q^{\prime}<2$ будем имееть

$$
\left\|w_{1}\right\|_{q^{\prime}} \leqslant\left\|v_{1}\right\|_{q^{\prime}}+\left\|u_{1}\right\|_{2} \leqslant\left\|v_{1}\right\|_{q^{\prime}}+\sqrt{M} .
$$

Далее, воспользовавшись теоремой Литтлвуда-Пэли и неравенством $|a+b|^{\alpha} \leqslant$ $|a|^{\alpha}+|b|^{\alpha}, 0 \leqslant \alpha \leqslant 1$, получим

$$
\begin{aligned}
\left\|v_{1}\right\|_{q^{\prime}} & \ll\left\|\left(\sum_{(s, 1) \leqslant l}\left|\delta_{s}\left(v_{1}, x\right)\right|^{2}\right)^{\frac{1}{2}}\right\|_{q^{\prime}}=\left\|\left(\sum_{(s, 1) \leqslant l}\left|\delta_{s}^{+}\left(v_{1}, x\right)\right|^{2}\right)^{\frac{q^{\prime}}{2}}\right\|_{1}^{\frac{1}{q^{\prime}}} \\
& \leqslant\left\|\sum_{(s, 1) \leqslant l}\left|\delta_{s}^{+}\left(v_{1}, x\right)\right|^{q^{\prime}}\right\|_{1}^{\frac{1}{q}}=\left(\sum_{(s, 1) \leqslant l}\left\|\delta_{s}^{+}\left(v_{1}, x\right)\right\|_{q^{\prime}}^{q^{\prime}}\right)^{\frac{1}{q^{\prime}}} \\
& \asymp\left(\sum_{(s, 1) \leqslant l} 2^{(s, 1)\left(1-\frac{1}{q^{\prime}}\right) q^{\prime}}\right)^{\frac{1}{q^{\prime}}} \leqslant 2^{\frac{l}{q}}\left(\sum_{(s, 1)=l} 1\right)^{\frac{1}{q^{\prime}}} \asymp 2^{\frac{l}{q}} l^{\frac{d-1}{q^{\prime}}} .
\end{aligned}
$$

Таким образом, с учетом значения $l$, из $(2.52)$ и (2.51) приходим к оценке

$$
\left\|w_{1}\right\|_{q^{\prime}} \ll 2^{\frac{l}{q}} l^{\frac{d-1}{q^{\prime}}}+\sqrt{M} \ll 2^{\frac{l}{q}} l^{\frac{d-1}{q^{\prime}}} .
$$

Следовательно, функция $P_{1}(x)=C_{2} 2^{-\frac{l}{q}} l^{-\frac{d-1}{q^{\prime}}} w_{1}(x)$ с некоторой постоянной $C_{2}>0$ удовлетворяет требованиям, предъявляемым к $P(x)$ из соотношения $(2.46)$. 
Подставив функции $f_{1}(x)$ и $P_{1}(x)$ в $(2.46)$, получим

$$
e_{M}\left(f_{1}\right)_{q} \gg\left(\sum_{l_{1}<(s, 1) \leqslant l} \sum_{k \in \rho^{+}(s)} \prod_{j=1}^{d} k_{j}^{-1}\right) 2^{-\frac{l}{q}} l^{-\frac{d-1}{q^{\prime}}} l^{-\frac{d}{\theta}}
$$

где $l_{1}$ - число, удовлетворяющее условию $2^{l_{1}} l_{1}^{d-1} \asymp M$. Далее, поскольку

$$
\begin{aligned}
\sum_{l_{1}<(s, 1) \leqslant l} \sum_{k \in \rho^{+}(s)} \prod_{j=1}^{d} k_{j}^{-1} & =\sum_{l_{1}<(s, 1) \leqslant l} \prod_{j=1}^{d} \sum_{k_{j}=2^{s_{j}-1}}^{2^{s_{j}}-1} k_{j}^{-1} \asymp \sum_{l_{1}<(s, 1) \leqslant l} 1 \\
& =\sum_{i=l_{1}+1}^{l} \sum_{(s, 1)=i} 1 \asymp \sum_{i=l_{1}+1}^{l} i^{d-1} \gg l_{1}^{d},
\end{aligned}
$$

то согласно (2.53) будем иметь

$$
e_{M}\left(f_{1}\right)_{q} \gg 2^{-\frac{l}{q}} l^{-\frac{d-1}{q^{\prime}}} l_{1}^{\frac{d}{\theta^{\prime}}} \asymp M^{-\frac{1}{2}}\left(\log ^{d} M\right)^{\frac{1}{\theta^{\prime}}},
$$

что и требовалось доказать.

Пусть $r_{1}>\frac{1}{p}$. Заметим, что в этом случае искомую оценку снизу достаточно получить при $q=2$. По заданному $M$ подберем $l$ таким, чтобы выполнялись соотношения $M \asymp 2^{l} l^{d-1}$ и $2^{l} l^{d-1} \geqslant 2 M$, и рассмотрим функцию

$$
f_{2}(x)=C_{3} 2^{-l\left(1-\frac{1}{p}\right)} l^{-\frac{d-1}{\theta}} \sum_{l \leqslant(s, 1) \leqslant l+d} \sum_{k \in \rho^{+}(s)} \prod_{j=1}^{d} k_{j}^{-r_{1}} \cos k_{j} x_{j}, \quad C_{3}>0 .
$$

Пусть сначала $p=1$. Тогда поскольку функция

$$
F_{r}(x)=\sum_{k>0} \prod_{j=1}^{d} k_{j}^{-r_{1}} \cos k_{j} x_{j}
$$

принадлежит классу $b H_{1}^{r}, b>0$ (см., например, [3, с. 39]), то согласно теореме В $\left\|A_{s}\left(F_{r}, x\right)\right\|_{1} \ll 2^{-(s, r)}$. Воспользовавшись этим неравенством, нетрудно проверить, что функция

$$
f_{2}(x)=C_{3} l^{-\frac{d-1}{\theta}} \sum_{l \leqslant(s, 1) \leqslant l+d} \sum_{k \in \rho^{+}(s)} \prod_{j=1}^{d} k_{j}^{-r_{1}} \cos k_{j} x_{j}, \quad C_{3}>0
$$

принадлежит классу $B_{1, \theta}^{r}$.

Если же $p>1$, то поступим следуюшим образом. Запишем $f_{2}(x)$ в виде

$$
f_{2}(x)=C_{3} 2^{-l\left(1-\frac{1}{p}\right)} l^{-\frac{d-1}{\theta}} g_{l}(x), \quad C_{3}>0,
$$


и для оценки $\left\|g_{l}\right\|_{B_{p, \theta}^{r}}$ воспользуемся неравенством Бернштейна (см. [3, с. 14]). Поскольку

$$
\begin{aligned}
\left\|\delta_{s}^{+}\left(g_{l}, x\right)\right\|_{p} & =\left\|\prod_{j=1}^{d} \sum_{k_{j} \in \rho^{+}\left(s_{j}\right)} k_{j}^{-r_{1}} \cos k_{j} x_{j}\right\|_{p} \ll \prod_{j=1}^{d} 2^{-s_{j} r_{1}}\left\|_{k_{j} \in \rho^{+}\left(s_{j}\right)} \cos k_{j} x_{j}\right\|_{p} \\
& \asymp \prod_{j=1}^{d} 2^{-s_{j} r_{1}} 2^{s_{j}\left(1-\frac{1}{p}\right)}=2^{-(s, 1)\left(r_{1}-1+\frac{1}{p}\right)}
\end{aligned}
$$

то

$$
\begin{aligned}
\left\|g_{l}\right\|_{B_{p, \theta}^{r}} & \asymp\left(\sum_{l \leqslant(s, 1) \leqslant l+d} 2^{(s, r) \theta}\left\|\delta_{s}^{+}\left(g_{l}, x\right)\right\|_{p}^{\theta}\right)^{\frac{1}{\theta}} \\
& \ll\left(\sum_{l \leqslant(s, 1) \leqslant l+d} 2^{(s, 1) r_{1} \theta} 2^{-(s, 1)\left(r_{1}-1+\frac{1}{p}\right) \theta}\right)^{\frac{1}{\theta}} \\
& =\left(\sum_{l \leqslant(s, 1) \leqslant l+d} 2^{(s, 1)\left(1-\frac{1}{p}\right) \theta}\right)^{\frac{1}{\theta}} \asymp 2^{l\left(1-\frac{1}{p}\right)} l^{\frac{d-1}{\theta}}
\end{aligned}
$$

при $1 \leqslant \theta<\infty$ и

$$
\begin{aligned}
\left\|g_{l}\right\|_{B_{p, \infty}^{r}} & =\sup _{\substack{s: \\
l \leqslant(s, 1) \leqslant l+d}} 2^{(s, r)}\left\|\delta_{s}^{+}\left(g_{l}, x\right)\right\|_{p} \\
& \ll \sup _{\substack{s: \\
l \leqslant(s, 1) \leqslant l+d}} 2^{(s, 1) r_{1}} 2^{-(s, 1)\left(r_{1}-1+\frac{1}{p}\right)} \asymp 2^{l\left(1-\frac{1}{p}\right)}
\end{aligned}
$$

при $\theta=\infty$.

Таким образом, согласно $(2.57)$ и (2.56) функция $f_{2}(x)$, определенная равенством $(2.55)$, принадлежит классу $B_{p, \theta}^{r}, 1 \leqslant \theta \leqslant \infty, 1<p<\infty$.

Представим функцию $P(x)$ из соотношения (2.46) как функцию

$$
P_{2}(x)=C_{4} 2^{-\frac{l}{2}} l^{-\frac{d-1}{2}} w_{1}(x), \quad C_{4}>0
$$

которая, как легко проверить, при $q=2$ удовлетворяет предъявляемым к ней требованиям. 
Подставив функции из (2.58) и (2.55) в (2.46), будем иметь

$$
\begin{aligned}
e_{M}\left(f_{2}\right)_{2} & \gg 2^{-l\left(1-\frac{1}{p}\right)} l^{-\frac{d-1}{\theta}} 2^{-\frac{l}{2}} l^{-\frac{d-1}{2}}\left(\sum_{(s, 1)=l+d} \sum_{k \in \rho^{+}(s)} \prod_{j=1}^{d} k_{j}^{-r_{1}}\right) \\
& =2^{-l\left(1-\frac{1}{p}+\frac{1}{2}\right)} l^{-(d-1)\left(\frac{1}{2}+\frac{1}{\theta}\right)}\left(\sum_{(s, 1)=l+d} \prod_{j=1}^{d} \sum_{k_{j}=2^{s_{j}-1}}^{2^{s_{j}-1}} k_{j}^{-r_{1}}\right) \\
& \gg 2^{-l\left(1-\frac{1}{p}+\frac{1}{2}\right)} l^{-(d-1)\left(\frac{1}{2}+\frac{1}{\theta}\right)}\left(\sum_{(s, 1)=l+d} \prod_{j=1}^{d} 2^{s_{j}\left(1-r_{1}\right)}\right) \\
& \asymp 2^{-l\left(1-\frac{1}{p}+\frac{1}{2}\right)} l^{-(d-1)\left(\frac{1}{2}+\frac{1}{\theta}\right)} \sum_{(s, 1)=l+d} 2^{-(s, 1)\left(r_{1}-1\right)} \\
& \asymp 2^{-l\left(1-\frac{1}{p}+\frac{1}{2}\right)} l^{-(d-1)\left(\frac{1}{2}+\frac{1}{\theta}\right)} 2^{-l\left(r_{1}-1\right)} l^{d-1} \\
& =2^{-l\left(r_{1}-\frac{1}{p}+\frac{1}{2}\right)} l^{(d-1)\left(\frac{1}{2}-\frac{1}{\theta}\right)} \asymp\left(M^{-1} \log ^{d-1} M\right)^{r_{1}-\frac{1}{p}+\frac{1}{2}}\left(\log ^{d-1} M\right)^{\frac{1}{2}-\frac{1}{\theta}} .
\end{aligned}
$$

Отсюда следует требуемая оценка снизу $e_{M}\left(B_{p, \theta}^{r}\right)_{q}$ в случае $r_{1}>\frac{1}{p}$.

Для завершения доказательства теоремы осталось получить оценку снизу $e_{M}\left(B_{p, \theta}^{r}\right)_{q}$ в случае $\frac{1}{p}-\frac{1}{q}<r_{1}<\frac{1}{p}$.

Аналогично, как и в случае $r_{1}=\frac{1}{p}$, будем рассматривать функцию $f_{2}(x)$ (см. (2.55)), где число $l$, фигурируюшее в ее определении, найдем из соотношения

$$
l=\frac{q}{2} \log M-(d-1)(q-1) \log \log M .
$$

Тогда, взяв в качестве $P(x)$ функцию

$$
P_{3}(x)=C_{5} 2^{-\frac{l}{q}} l^{-\frac{d-1}{q^{\prime}}} w_{1}(x), \quad C_{5}>0,
$$

и воспользовавшись соотношением (2.46), получим

$$
\begin{aligned}
e_{M}\left(f_{2}\right)_{q} & \gg 2^{-l\left(1-\frac{1}{p}+\frac{1}{q}\right)} l^{-(d-1)\left(\frac{1}{\theta}+\frac{1}{q^{\prime}}\right)}\left(\sum_{(s, 1)=l+d} \sum_{k \in \rho^{+}(s)} \prod_{j=1}^{d} k_{j}^{-r_{1}}\right) \\
& \gg 2^{-l\left(1-\frac{1}{p}+\frac{1}{q}\right)} l^{-(d-1)\left(\frac{1}{\theta}+\frac{1}{q^{\prime}}\right)} 2^{-l\left(r_{1}-1\right)} l^{d-1} \\
& =2^{-l\left(r_{1}-\frac{1}{p}+\frac{1}{q}\right)} l^{(d-1)\left(\frac{1}{q}-\frac{1}{\theta}\right)} \asymp M^{-\frac{q}{2}\left(r_{1}-\frac{1}{p}+\frac{1}{q}\right)}\left(\log ^{d-1} M\right)^{(q-1)\left(r_{1}-\frac{1}{p}+\frac{q^{\prime}}{q \theta^{\prime}}\right)} .
\end{aligned}
$$

Обратим внимание, что полученная оценка совпадает по порядку с оценкой сверху при $r_{1} \geqslant \frac{1}{p}-\frac{q^{\prime}}{q \theta^{\prime}}$. Если же $\frac{1}{p}-\frac{1}{q}<r_{1}<\frac{1}{p}-\frac{q^{\prime}}{q \theta^{\prime}}$, то заметим следующее. $\mathrm{C}$ одной стороны, установленная выше оценка сверху $e_{M}\left(B_{p, \theta}^{r}\right)_{q}$ в этом случае не зависит от размерности пространства $\mathbb{R}^{d}$, значит, требуемую оценку снизу достаточно установить при $d=1$. С другой стороны, в одномерном случае проведенные вьше рассуждения позволяют получить нужную оценку для всех $r_{1}>\frac{1}{p}-\frac{1}{q}$. 
Теорема доказана полностью.

Отметим, что при $1<p \leqslant 2<q<\infty$ порядки величин $e_{M}\left(H_{p}^{r}\right)_{q}$ при всех возможных значениях параметра $r_{1}$, а также величин $e_{M}\left(W_{p, \alpha}^{r}\right)_{q}$, кроме случая $\frac{1}{p}-\frac{1}{q}<r_{1}<\frac{1}{p}$, анонсированы в [15]. Порядок $e_{M}\left(W_{p, \alpha}^{r}\right)_{q}, 1<p \leqslant 2<q<\infty$, при $\frac{1}{p}-\frac{1}{q}<r_{1}<\frac{1}{p}$ был установлен в [16]. Точные порядки величин $e_{M}\left(H_{1}^{r}\right)_{q}$ и $e_{M}\left(W_{1, \alpha}^{r}\right)_{q}$ при $2<q<\infty$ легко получить как следствия соответствующих оценок приближений ядер Бернулли $F_{r}(x, \alpha)$ и тригонометрических поперечников классов $H_{1}^{r}$. Эти результаты также анонсированы в [14].

Теперь перейдем к рассмотрению случая $2 \leqslant p \leqslant q<\infty$.

Справедливо следующее утверждение.

Теорема 2.2. Пусть $2 \leqslant p \leqslant q<\infty, 1 \leqslant \theta \leqslant \infty$. Тогда при $r_{1}>\frac{1}{2}$ имеет место порядковая оченка

$$
e_{M}\left(B_{p, \theta}^{r}\right)_{q} \asymp M^{-r_{1}}\left(\log ^{\nu-1} M\right)^{r_{1}+\frac{1}{2}-\frac{1}{\theta}} .
$$

ДокАЗАТЕЛЬСтво. Оценка сверху следует из теоремы 2.1 при $p=2$ согласно включению $B_{p, \theta}^{r} \subset B_{2, \theta}^{r}, p \geqslant 2$. При нахождении оценки снизу будем пользоваться известным результатом Рудина-Шапиро (см., например, [35, с. 155]): для каждого $m \in \mathbb{N}$ найдется полином

$$
R_{m}(x)=\sum_{j=2^{m-1}}^{2^{m}-1} \varepsilon_{j} e^{i j x}, \quad \varepsilon_{j}= \pm 1
$$

такой, что $\left\|R_{m}\right\|_{\infty} \ll 2^{\frac{m}{2}}$.

Итак, выберем по заданному $M$ число $l$ таким, чтобы выполнялись соотношения $2^{l} l^{d-1} \asymp M, 2^{l} l^{d-1} \geqslant 2 M$, и рассмотрим функцию

$$
f_{3}(x)=C_{6} 2^{-l\left(r_{1}+\frac{1}{2}\right)} l^{-\frac{d-1}{\theta}} \sum_{(s, 1) \leqslant l} \prod_{j=1}^{d} R_{s_{j}}\left(x_{j}\right), \quad C_{6}>0
$$

Поскольку

$$
\delta_{s}\left(f_{3}, x\right)=C_{6} 2^{-l\left(r_{1}+\frac{1}{2}\right)} l^{-\frac{d-1}{\theta}} \prod_{j=1}^{d} R_{s_{j}}\left(x_{j}\right)
$$

то легко убедиться, что функция $f_{3}(x)$, с соответствующей постоянной $C_{6}>0$, принадлежит классу $B_{p, \theta}^{r}, 1 \leqslant \theta \leqslant \infty$.

Функцию $P_{4}(x)$, которая удовлетворяла бы требованиям, налагаемым на функцию $P(x)$ из $(2.46)$, построим следуюшим образом. Пусть

$$
v_{2}(x)=\sum_{(s, 1) \leqslant l} \prod_{j=1}^{d} R_{s_{j}}\left(x_{j}\right), \quad u_{2}(x)=\sum_{(s, 1) \leqslant l}^{*} \prod_{j=1}^{d} R_{s_{j}}\left(x_{j}\right),
$$


где "звездочка" означает, что полином $u_{2}(x)$ содержит только те гармоники функции $v_{2}(x)$, которые имеют "номера" из множества $\Omega_{M}$. Тогда, обозначив $w_{2}(x)=$ $v_{2}(x)-u_{2}(x)$, будем иметь

$$
\left\|w_{2}\right\|_{q^{\prime}} \leqslant\left\|w_{2}\right\|_{2} \leqslant\left\|v_{2}\right\|_{2}+\left\|u_{2}\right\|_{2} \ll 2^{\frac{l}{2} l^{\frac{d-1}{2}}} .
$$

Из этого соотношения заключаем, что функция

$$
P_{4}(x)=C_{7} 2^{-\frac{l}{2}} l^{-\frac{d-1}{2}} w_{2}(x), \quad C_{7}>0
$$

удовлетворяет предъявляемым к ней требованиям. Таким образом, подставив $f_{3}(x)$ и $P_{4}(x)$ в $(2.46)$, будем иметь

$$
\begin{aligned}
e_{M}\left(f_{3}\right)_{q} & \gg 2^{-l\left(r_{1}+1\right)} l^{-(d-1)\left(\frac{1}{2}+\frac{1}{\theta}\right)}\left(2^{l} l^{d-1}-M\right) \\
& \gg 2^{-l\left(r_{1}+1\right)} l^{-(d-1)\left(\frac{1}{2}+\frac{1}{\theta}\right)} 2^{l} l^{d-1}=2^{-l r_{1}} l^{(d-1)\left(\frac{1}{2}-\frac{1}{\theta}\right)} \\
& \asymp M^{-r_{1}}\left(\log ^{d-1} M\right)^{r_{1}+\frac{1}{2}-\frac{1}{\theta}} .
\end{aligned}
$$

Оценка снизу, а вместе с ней и теорема доказаны.

Отметим, что с помошю аналогичных рассуждений нетрудно показать, что для классов $W_{p, \alpha}^{r}$ имеет место соотношение

$$
e_{M}\left(W_{p, \alpha}^{r}\right)_{q} \asymp M^{-r_{1}}\left(\log ^{\nu-1} M\right)^{r_{1}}
$$

при $r_{1}>\frac{1}{2}, 2 \leqslant p \leqslant q<\infty$.

$$
\begin{gathered}
\S \text { 3. Порядки } e_{M}\left(B_{p, \theta}^{r}\right)_{q} \text { при } 1 \leqslant p \leqslant q \leqslant 2,(p, q) \neq(1,1) \\
\text { и } 1<q<p<\infty, p \geqslant 2
\end{gathered}
$$

TЕорема 3.1. Пусть $1 \leqslant p \leqslant q \leqslant 2,(p, q) \neq(1,1), u r_{1}>\frac{1}{p}-\frac{1}{q}$. Тогда прu $1 \leqslant \theta \leqslant \infty$

$$
e_{M}\left(B_{p, \theta}^{r}\right)_{q} \asymp M^{-\left(r_{1}-\frac{1}{p}+\frac{1}{q}\right)}\left(\log ^{\nu-1} M\right)^{\left(r_{1}-\frac{1}{p}+\frac{2}{q}-\frac{1}{\theta}\right)_{+}} .
$$

ДоказАтЕльство. Сначала установим в (3.1) оценку сверху в случае $1 \leqslant p<$ $q \leqslant 2$. Покажем, что если $\theta \geqslant q$, то требуемая оценка следует из оценки приближения функций $f(x)$ из класса $B_{p, \theta}^{r}$ их ступенчатыми гиперболическими суммами Фурье

$$
S_{n}^{\gamma}(f, x)=\sum_{(s, \gamma)<n} \delta_{s}(f, x), \quad M \asymp 2^{n} n^{\nu-1} .
$$


Действительно, пусть $q_{0}$ - произвольное число, удовлетворяющее условию $p<$ $q_{0}<q$. В таком случае, согласно лемме А и теореме Б, для $f \in B_{p, \theta}^{r}$ будем иметь

$$
\begin{aligned}
\left\|f(x)-S_{n}^{\gamma}(f, x)\right\|_{q} & =\left\|\sum_{(s, \gamma) \geqslant n} \delta_{s}(f, x)\right\|_{q} \\
& \ll\left\{\sum_{(s, \gamma) \geqslant n}\left(\left\|\delta_{s}(f, x)\right\|_{q_{0}} 2^{\|s\|_{1}\left(\frac{1}{q_{0}}-\frac{1}{q}\right)}\right)^{q}\right\}^{\frac{1}{q}} \\
& \asymp\left\{\sum_{(s, \gamma) \geqslant n}\left(\left\|A_{s}(f, x)\right\|_{q_{0}} 2^{\|s\|_{1}\left(\frac{1}{q_{0}}-\frac{1}{q}\right)}\right)^{q}\right\}^{\frac{1}{q}} \\
& =\left\{\sum_{(s, \gamma) \geqslant n}\left(\left\|A_{s}(f, x)\right\|_{p} 2^{\|s\|_{1}\left(\frac{1}{p}-\frac{1}{q}\right)}\right)^{q}\right\}^{\frac{1}{q}} .
\end{aligned}
$$

Пусть $q<\theta<\infty$. Тогда, применив к последней сумме неравенство Гёльдера с показателем $\theta / q$, продолжим оценку (3.2):

$$
\begin{aligned}
& \leqslant\left(\sum_{(s, \gamma) \geqslant n} 2^{(s, r) \theta}\left\|A_{s}(f, x)\right\|_{p}^{\theta}\right)^{\frac{1}{\theta}}\left(\sum_{(s, \gamma) \geqslant n} 2^{-\left((s, r)-\|s\|_{1}\left(\frac{1}{p}-\frac{1}{q}\right)\right) \frac{\theta q}{\theta-q}}\right)^{\frac{1}{q}-\frac{1}{\theta}} \\
& \ll\|f\|_{B_{p, \theta}^{r}}\left(\sum_{(s, \gamma) \geqslant n} 2^{-\left(s, r-\frac{1}{p}+\frac{1}{q}\right) \frac{\theta q}{\theta-q}}\right)^{\frac{1}{q}-\frac{1}{\theta}} \leqslant\left(\sum_{(s, \gamma) \geqslant n} 2^{-(s, \bar{\gamma})\left(r_{1}-\frac{1}{p}+\frac{1}{q}\right) \frac{\theta q}{\theta-q}}\right)^{\frac{1}{q}-\frac{1}{\theta}},
\end{aligned}
$$

где $r-\frac{1}{p}+\frac{1}{q}-$ вектор с координатами $r_{j}-\frac{1}{p}+\frac{1}{q}, j=\overline{1, d}$, a $\bar{\gamma}=\left(\bar{\gamma}_{1}, \ldots, \bar{\gamma}_{d}\right)-$ вектор с координатами $\bar{\gamma}_{j}=\left(r_{j}-\frac{1}{p}+\frac{1}{q}\right) /\left(r_{1}-\frac{1}{p}+\frac{1}{q}\right), j=\overline{1, d}$.

Поскольку $\bar{\gamma}_{j}=\gamma_{j}, j=\overline{1, \nu}$, и $\gamma_{j}<\bar{\gamma}_{j}, j=\overline{\nu+1, d}$, то, воспользовавшись для оценки последней суммы в (3.3) соотношением (1.3), получим

$$
\left\|f(x)-S_{n}^{\gamma}(f, x)\right\|_{q} \ll 2^{-n\left(r_{1}-\frac{1}{p}+\frac{1}{q}\right)} n^{(\nu-1)\left(\frac{1}{q}-\frac{1}{\theta}\right)} .
$$

Отсюда в силу того, что $M \asymp 2^{n} n^{\nu-1}$, будем иметь при $q<\theta<\infty$

$$
e_{M}\left(B_{p, \theta}^{r}\right)_{q} \ll M^{-\left(r_{1}-\frac{1}{p}+\frac{1}{q}\right)}\left(\log ^{\nu-1} M\right)^{r_{1}-\frac{1}{p}+\frac{2}{q}-\frac{1}{\theta}} .
$$

Из (3.2) также легко получить нужную оценку и при $\theta=q$ :

$$
e_{M}\left(B_{p, \theta}^{r}\right)_{q} \ll M^{-\left(r_{1}-\frac{1}{p}+\frac{1}{q}\right)}\left(\log ^{\nu-1} M\right)^{r_{1}-\frac{1}{p}+\frac{1}{q}} .
$$

Если же $\theta=\infty$, то $\left\|A_{s}(f, x)\right\|_{p} \ll 2^{-(s, r)}$, и, следовательно, из оценки (3.2) находим

$$
\begin{aligned}
e_{M}\left(B_{p, \theta}^{r}\right)_{q} & \ll\left\{\sum_{(s, \gamma) \geqslant n}\left(2^{-(s, r)} 2^{\|s\|_{1}\left(\frac{1}{p}-\frac{1}{q}\right)}\right)^{q}\right\}^{\frac{1}{q}} \\
& \leqslant\left(\sum_{(s, \gamma) \geqslant n} 2^{-\left(s, r-\frac{1}{p}+\frac{1}{q}\right) q}\right)^{\frac{1}{q}}=\left(\sum_{(s, \gamma) \geqslant n} 2^{-(s, \bar{\gamma})\left(r_{1}-\frac{1}{p}+\frac{1}{q}\right) q}\right)^{\frac{1}{q}} \\
& \ll 2^{-n\left(r_{1}-\frac{1}{p}+\frac{1}{q}\right)} n^{\frac{\nu-1}{q}} \asymp M^{-\left(r_{1}-\frac{1}{p}+\frac{1}{q}\right)}\left(\log ^{\nu-1} M\right)^{r_{1}-\frac{1}{p}+\frac{2}{q}} .
\end{aligned}
$$


Теперь остановимся на получении оценки сверху величины $e_{M}\left(B_{p, \theta}^{r}\right)_{q}$ в случае $1 \leqslant \theta<q$. Построим для $f \in B_{p, \theta}^{r}$ приближаюший полином $P\left(\Omega_{M}, x\right)$, которьй доставляет требуемую оценку приближения. Пусть $n$ удовлетворяет соотношению $M \asymp 2^{n} n^{\nu-1}$ и $n_{0}=n+(\nu-1) \log n$. Полином $P\left(\Omega_{M}, x\right)$ будем строить в виде

$$
P\left(\Omega_{M}, x\right)=R(x)+Q(x),
$$

где $R(x)=\sum_{(s, \gamma)<n} A_{s}(f, x)$, а слагаемое $Q(x)$ построим в процессе нахождения оценки.

Для натурального $l$ по-прежнему положим

$$
S_{l}=\left(\sum_{l \leqslant(s, \gamma)<l+1}\left\|A_{s}(f, x)\right\|_{p}^{\theta} 2^{(s, r) \theta}\right)^{\frac{1}{\theta}}
$$

и пусть $m_{l}^{*}=\left[2^{n} n^{\nu-1} 2^{-l} S_{l}^{\theta}\right]+1$. Рассмотрим полином

$$
R^{*}(x)=\sum_{l=n}^{\left[n_{0}\right]+1} \sum_{l \leqslant(s, \gamma)<l+1} A_{s}(f, x)
$$

и каждому $l \in\left[n, n_{0}\right), l \in \mathbb{N}$, сопоставим из внутренней суммы $(3.4) m_{l}^{*}$ "блоков" $A_{s}(f, x)$ с наибольшими значениями $\left\|A_{s}(f, x)\right\|_{p}$. Расположим эти значения в порядке убывания и обозначим их $\alpha_{i}(f, l), i=\overline{1, m_{l}^{*}}$. Совокупность таким образом полученных "блоков" $A_{s}(f, x)$ по всем $l \in\left[n, n_{0}\right), l \in \mathbb{N}$, обозначим $Q(x)$.

Покажем, что количество гармоник $K$, которые образуют полином $P\left(\Omega_{M}, x\right)$, не превьшает по порядку $M$. Действительно,

$$
\begin{aligned}
K & \ll 2^{n} n^{\nu-1}+\sum_{l=n}^{\left[n_{0}\right]+1} 2^{l} m_{l}^{*} \\
& \ll 2^{n} n^{\nu-1}+2^{n} n^{\nu-1} \sum_{l=n}^{\left[n_{0}\right]+1} \sum_{l \leqslant(s, \gamma)<l+1} 2^{(s, r) \theta}\left\|A_{s}(f, x)\right\|_{p}^{\theta} \\
& \ll 2^{n} n^{\nu-1}+2^{n} n^{\nu-1}\|f\|_{B_{p, \theta}^{r}}^{\theta}=2^{n} n^{\nu-1}\left(1+\|f\|_{B_{p, \theta}^{r}}^{\theta}\right) \ll 2^{n} n^{\nu-1} \asymp M .
\end{aligned}
$$

Получим теперь оценку сверху для $\left\|f(x)-P\left(\Omega_{M}, x\right)\right\|_{q}$. Имеем

$$
\begin{aligned}
\left\|f(x)-P\left(\Omega_{M}, x\right)\right\|_{q} & =\|f(x)-R(x)-Q(x)\|_{q} \\
& =\left\|f(x)-\sum_{(s, \gamma)<n_{0}} A_{s}(f, x)+R^{*}(x)-Q(x)\right\|_{q} \\
& \leqslant\left\|f(x)-\sum_{(s, \gamma)<n_{0}} A_{s}(f, x)\right\|_{q}+\left\|R^{*}(x)-Q(x)\right\|_{q}=I_{7}+I_{8} .
\end{aligned}
$$


Далее, согласно лемме А и теореме Б, имеем

$$
\begin{aligned}
I_{7} & \ll\left\{\sum_{(s, \gamma) \geqslant n_{0}}\left(\left\|A_{s}(f, x)\right\|_{p} 2^{\|s\|_{1}\left(\frac{1}{p}-\frac{1}{q}\right)}\right)^{q}\right\}^{\frac{1}{q}} \\
& \leqslant\left(\sum_{(s, \gamma) \geqslant n_{0}}\left\|A_{s}(f, x)\right\|_{p}^{\theta} 2^{\|s\|_{1}\left(\frac{1}{p}-\frac{1}{q}\right) \theta}\right)^{\frac{1}{\theta}} \\
& \leqslant\left(\sum_{(s, \gamma) \geqslant n_{0}} 2^{(s, r) \theta}\left\|A_{s}(f, x)\right\|_{p}^{\theta} 2^{-(s, \gamma)\left(r_{1}-\frac{1}{p}+\frac{1}{q}\right) \theta}\right)^{\frac{1}{\theta}} \\
& \leqslant 2^{-n_{0}\left(r_{1}-\frac{1}{p}+\frac{1}{q}\right)}\|f\|_{B_{p, \theta}^{r}} \leqslant 2^{-n_{0}\left(r_{1}-\frac{1}{p}+\frac{1}{q}\right)} .
\end{aligned}
$$

Принимая во внимание, что $n_{0}=n+(\nu-1) \log n$, из (3.6) получаем оценку

$$
I_{7} \ll 2^{-n\left(r_{1}-\frac{1}{p}+\frac{1}{q}\right)} n^{-(\nu-1)\left(r_{1}-\frac{1}{p}+\frac{1}{q}\right)} \asymp M^{-\left(r_{1}-\frac{1}{p}+\frac{1}{q}\right)} .
$$

Оценим $I_{8}$. Согласно лемме А и принятым обозначениям имеем

$$
\begin{aligned}
I_{8} \ll & \left(\sum_{l=n}^{\left[n_{0}\right]+1} \sum_{i>m_{l}^{*}} \alpha_{i}^{q}(f, l) 2^{l\left(\frac{1}{p}-\frac{1}{q}\right) q}\right)^{\frac{1}{q}} \\
= & \left(\sum_{l=n}^{\left[n_{0}\right]+1} \sum_{i>m_{l}^{*}} \alpha_{i}^{\theta}(f, l) \alpha_{i}^{q-\theta}(f, l) 2^{l\left(\frac{1}{p}-\frac{1}{q}\right) q}\right)^{\frac{1}{q}} \\
\ll & \left(\sum_{l=n}^{\left[n_{0}\right]+1} m_{l}^{*-\frac{q-\theta}{\theta}} 2^{-l(q-\theta) r_{1}} S_{l}^{q-\theta} 2^{-l \theta r_{1}} 2^{l\left(\frac{1}{p}-\frac{1}{q}\right) q}\right. \\
& \left.\times \sum_{l \leqslant(s, \gamma)<l+1} 2^{(s, r) \theta}\left\|A_{s}(f, x)\right\|_{p}^{\theta}\right)^{\frac{1}{q}} \\
\ll & \left(\sum_{l=n}^{\left[n_{0}\right]+1} m_{l}^{*-\frac{q-\theta}{\theta}} 2^{-l\left(r_{1}-\frac{1}{p}+\frac{1}{q}\right) q} S_{l}^{q-\theta} S_{l}^{\theta}\right)^{\frac{1}{q}} \\
= & \left(\sum_{l=n}^{\left[n_{0}\right]+1} m_{l}^{*-\frac{q-\theta}{\theta}} 2^{-l\left(r_{1}-\frac{1}{p}+\frac{1}{q}\right) q} S_{l}^{q}\right)^{\frac{1}{q}} .
\end{aligned}
$$

Подставив вместо $m_{l}^{*}$ их значения, продолжим оценку (3.8):

$$
\begin{aligned}
& \ll\left(2^{n} n^{\nu-1}\right)^{\frac{1}{q}-\frac{1}{\theta}}\left(\sum_{l=n}^{\left[n_{0}\right]+1} S_{l}^{\theta} 2^{-l\left(r_{1}-\frac{1}{p}+\frac{1}{q}\right) q} 2^{l q\left(\frac{1}{\theta}-\frac{1}{q}\right)}\right)^{\frac{1}{q}} \\
& =\left(2^{n} n^{\nu-1}\right)^{\frac{1}{q}-\frac{1}{\theta}}\left(\sum_{l=n}^{\left[n_{0}\right]+1} 2^{-l q\left(r_{1}-\frac{1}{p}+\frac{2}{q}-\frac{1}{\theta}\right)} S_{l}^{\theta}\right)^{\frac{1}{q}}=\left(2^{n} n^{\nu-1}\right)^{\frac{1}{q}-\frac{1}{\theta}} I_{9} .
\end{aligned}
$$


При последуюшей оценке $I_{9}$ будем рассматривать два случая.

Пусть $r_{1}>\frac{1}{p}-\frac{2}{q}+\frac{1}{\theta}$. Тогда

$$
\begin{aligned}
I_{9} & \leqslant 2^{-n\left(r_{1}-\frac{1}{p}+\frac{2}{q}-\frac{1}{\theta}\right)}\left(\sum_{l=n}^{\left[n_{0}\right]+1} S_{l}^{\theta}\right)^{\frac{1}{q}} \\
& \ll 2^{-n\left(r_{1}-\frac{1}{p}+\frac{2}{q}-\frac{1}{\theta}\right)}\|f\|_{B_{p, \theta}^{r}}^{\frac{\theta}{q}} \leqslant 2^{-n\left(r_{1}-\frac{1}{p}+\frac{2}{q}-\frac{1}{\theta}\right)} .
\end{aligned}
$$

Подставив (3.10) в (3.9), получим

$$
I_{8} \ll 2^{-n\left(r_{1}-\frac{1}{p}+\frac{1}{q}\right)} n^{(\nu-1)\left(\frac{1}{q}-\frac{1}{\theta}\right)} \asymp M^{-\left(r_{1}-\frac{1}{p}+\frac{1}{q}\right)}\left(\log ^{\nu-1} M\right)^{r_{1}-\frac{1}{p}+\frac{2}{q}-\frac{1}{\theta}} .
$$

В случае $\frac{1}{p}-\frac{1}{q}<r_{1} \leqslant \frac{1}{p}-\frac{2}{q}+\frac{1}{\theta}$ будем иметь

$$
I_{9} \ll 2^{-n_{0}\left(r_{1}-\frac{1}{p}+\frac{2}{q}-\frac{1}{\theta}\right)}\|f\|_{B_{p, \theta}^{r}}^{\frac{\theta}{q}} \leqslant 2^{-n_{0}\left(r_{1}-\frac{1}{p}+\frac{2}{q}-\frac{1}{\theta}\right)},
$$

и согласно $(3.9)$

$$
I_{8} \ll M^{-\left(r_{1}-\frac{1}{p}+\frac{1}{q}\right)} .
$$

Таким образом, принимая во внимание оценки $(3.12),(3.11)$ и $(3.7)$ и воспользовавшись (3.5), приходим к искомой оценке сверху величины $e_{M}\left(B_{p, \theta}^{r}\right)_{q}$ и в случае $1 \leqslant \theta<q$.

Для завершения оценки сверху осталось рассмотреть случай $1<p=q \leqslant 2$. Отметим, что при этом, с незначительньми изменениями, используется та же схема рассуждений, что и при $1<p<q \leqslant 2$.

Пусть $p \leqslant \theta \leqslant \infty$. Рассмотрим приближение $f \in B_{p, \theta}^{r}$ ступенчатыми гиперболическими суммами Фурье $S_{n}^{\gamma^{\prime}}(f, x)=\sum_{\left(s, \gamma^{\prime}\right)<n} \delta_{s}(f, x), M \asymp 2^{n} n^{\nu-1}$. Имеем

$$
\begin{aligned}
\left\|f(x)-S_{n}^{\gamma^{\prime}}(f, x)\right\|_{p} & \ll\left\|\left(\sum_{\left(s, \gamma^{\prime}\right) \geqslant n}\left|\delta_{s}(f, x)\right|^{2}\right)^{\frac{1}{2}}\right\|_{p} \\
& \ll\left(\sum_{\left(s, \gamma^{\prime}\right) \geqslant n}\left\|\delta_{s}(f, x)\right\|_{p}^{p}\right)^{\frac{1}{p}} .
\end{aligned}
$$

Предположим, что $p<\theta<\infty$. Тогда, применив к последней сумме в (3.13) неравенство Гёльдера с показателем $\theta / p$, продолжим оценку:

$$
\begin{aligned}
& \leqslant\left(\sum_{\left(s, \gamma^{\prime}\right) \geqslant n} 2^{(s, r) \theta}\left\|\delta_{s}(f, x)\right\|_{p}^{\theta}\right)^{\frac{1}{\theta}}\left(\sum_{\left(s, \gamma^{\prime}\right) \geqslant n} 2^{-p(s, r) \frac{\theta}{\theta-p}}\right)^{\frac{1}{p}-\frac{1}{\theta}} \\
& \ll\|f\|_{B_{p, \theta}^{r}} 2^{-r_{1} n} n^{(\nu-1)\left(\frac{1}{p}-\frac{1}{\theta}\right)} \leqslant 2^{-r_{1} n} n^{(\nu-1)\left(\frac{1}{p}-\frac{1}{\theta}\right)} .
\end{aligned}
$$

Отсюда

$$
e_{M}\left(B_{p, \theta}^{r}\right)_{p} \ll M^{-r_{1}}\left(\log ^{\nu-1} M\right)^{r_{1}+\frac{1}{p}-\frac{1}{\theta}} .
$$


Из (3.13) легко получить требуемую оценку и при $\theta=p$ :

$$
e_{M}\left(B_{p, \theta}^{r}\right)_{p} \ll M^{-r_{1}}\left(\log ^{\nu-1} M\right)^{r_{1}}
$$

Если же $\theta=\infty$, то, принимая во внимание, что в таком случае для $f \in B_{p, \infty}^{r}$ имеем $\left\|\delta_{s}(f, x)\right\|_{p} \ll 2^{-(s, r)}$, из (3.13) находим

$$
\begin{aligned}
e_{M}(f)_{p} & \ll\left\|f(x)-S_{n}^{\gamma^{\prime}}(f, x)\right\|_{p} \ll\left(\sum_{\left(s, \gamma^{\prime}\right) \geqslant n} 2^{-p(s, r)}\right)^{\frac{1}{p}} \ll 2^{-n r_{1}} n^{\frac{\nu-1}{p}} \\
& \asymp M^{-r_{1}}\left(\log ^{\nu-1} M\right)^{r_{1}+\frac{1}{p}}
\end{aligned}
$$

Требуемые оценки сверху в случае $p \leqslant \theta \leqslant \infty$ установлены.

Рассмотрим случай $1 \leqslant \theta<p$. Пусть $f \in B_{p, \theta}^{r}, \quad M \asymp 2^{n} n^{\nu-1}$ и $n_{0}=n+$ $(\nu-1) \log n$. Положим

$$
P_{1}\left(\Omega_{M}, x\right)=R_{1}(x)+Q_{1}(x),
$$

где $R_{1}(x)=\sum_{(s, \gamma)<n} \delta_{s}(f, x)$, а $Q_{1}(x)$ построим по той же схеме, что и $Q(x)$. Для натурального $l$ положим

$$
\widetilde{S}_{l}=\left(\sum_{l \leqslant(s, \gamma)<l+1}\left\|\delta_{s}(f, x)\right\|_{p}^{\theta} 2^{(s, r) \theta}\right)^{\frac{1}{\theta}}
$$

и пусть $\widetilde{m}_{l}=\left[2^{n} n^{\nu-1} 2^{-l} \widetilde{S}_{l}^{\theta}\right]+1$. Рассмотрим полином

$$
R_{1}^{*}(x)=\sum_{l=n}^{\left[n_{0}\right]+1} \sum_{l \leqslant(s, \gamma)<l+1} \delta_{s}(f, x)
$$

и каждому $l \in\left[n, n_{0}\right), l \in \mathbb{N}$, сопоставим из внутренней суммы $(3.14) \widetilde{m}_{l}$ "блоков" $\delta_{s}(f, x)$ с наибольшими значениями $\left\|\delta_{s}(f, x)\right\|_{p}$. Эти значения для каждого $l \in\left[n, n_{0}\right), l \in \mathbb{N}$, упорядочим в порядке убывания и обозначим $\tilde{\alpha}_{i}(f, l), i=\overline{1, \widetilde{m}_{l}}$. Совокупность таким образом полученных "блоков" $\delta_{s}(f, x)$ образует некоторый полином, который и обозначим $Q_{1}(x)$. Нетрудно проверить, что количество гармоник в полиноме $P_{1}\left(\Omega_{M}, x\right)$ в таком случае не превышает по порядку $M$.

Таким образом, для $f \in B_{p, \theta}^{r}$ будем иметь

$$
\begin{aligned}
\left\|f(x)-P_{1}\left(\Omega_{M}, x\right)\right\|_{p} \leqslant & \left\|f(x)-\sum_{(s, \gamma)<n_{0}} \delta_{s}(f, x)\right\|_{p} \\
& +\left\|R_{1}^{*}(x)-Q_{1}(x)\right\|_{p}=I_{10}+I_{11} .
\end{aligned}
$$


Применив для оценки $I_{10}$ теорему Литтлвуда-Пэли и воспользовавшись соотношением (2.5), получим

$$
\begin{aligned}
I_{10} & \ll\left(\sum_{(s, \gamma) \geqslant n_{0}}\left|\delta_{s}(f, x)\right|^{2}\right)^{\frac{1}{2}} \|_{p} \ll\left(\sum_{(s, \gamma) \geqslant n_{0}}\left\|\delta_{s}(f, x)\right\|_{p}^{p}\right)^{\frac{1}{p}} \\
& \leqslant\left(\sum_{(s, \gamma) \geqslant n_{0}}\left\|\delta_{s}(f, x)\right\|_{p}^{\theta}\right)^{\frac{1}{\theta}} \leqslant \sup _{(s, \gamma) \geqslant n_{0}} 2^{-(s, r)}\left(\sum_{(s, \gamma) \geqslant n_{0}} 2^{(s, r) \theta}\left\|\delta_{s}(f, x)\right\|_{p}^{\theta}\right)^{\frac{1}{\theta}} \\
& \ll 2^{-n_{0} r_{1}}\|f\|_{B_{p, \theta}^{r}} \leqslant 2^{-n_{0} r_{1}} \leqslant 2^{-n r_{1}} n^{-(\nu-1) r_{1}} \asymp M^{-r_{1}} .
\end{aligned}
$$

Переходя к оценке $I_{11}$, обозначим через $\mathscr{D}_{f}$ множество векторов $s=\left(s_{1}, \ldots, s_{d}\right)$, по которым "блоки" $\delta_{s}(f, x)$ содержатся в $R_{1}^{*}(x)-Q_{1}(x)$. Тогда согласно теореме Литтлвуда-Пэли и определению множества $\mathscr{D}_{f}$ будем иметь

$$
\begin{aligned}
I_{11} & =\left\|\sum_{s \in \mathscr{D}_{f}} \delta_{s}(f, x)\right\|_{p} \asymp\left\|\left(\sum_{s \in \mathscr{D}_{f}}\left|\delta_{s}(f, x)\right|^{2}\right)^{\frac{1}{2}}\right\|_{p} \\
& \leqslant\left(\sum_{s \in \mathscr{D}_{f}}\left\|\delta_{s}(f, x)\right\|_{p}^{p}\right)^{\frac{1}{p}} \leqslant\left(\sum_{l=n}^{\left[n_{0}\right]+1} \sum_{i>\widetilde{m}_{l}} \tilde{\alpha}_{i}^{p}(f, l)\right)^{\frac{1}{p}} .
\end{aligned}
$$

Поскольку в силу определения чисел $\tilde{\alpha}_{i}(f, l)$ имеет место неравенство

$$
\tilde{\alpha}_{i}(f, l) \leqslant i^{-\frac{1}{\theta}} 2^{-l r_{1}} \widetilde{S}_{l}
$$

то из (3.17) находим

$$
\begin{aligned}
I_{11} & \ll\left(\sum_{l=n}^{\left[n_{0}\right]+1} \sum_{i>\widetilde{m}_{l}} \tilde{\alpha}_{i}^{\theta}(f, l) \tilde{\alpha}_{i}^{p-\theta}(f, l)\right)^{\frac{1}{p}} \\
& \leqslant\left(\sum_{l=n}^{\left[n_{0}\right]+1} \widetilde{m}_{l}^{-\frac{p-\theta}{\theta}} 2^{-l(p-\theta) r_{1}} \widetilde{S}_{l}^{p-\theta} 2^{-l \theta r_{1}} \sum_{l \leqslant(s, \gamma)<l+1} 2^{(s, r) \theta}\left\|\delta_{s}(f, x)\right\|_{p}^{\theta}\right)^{\frac{1}{p}} \\
& =\left(\sum_{l=n}^{\left[n_{0}\right]+1} \widetilde{m}_{l}^{-\frac{p-\theta}{\theta}} 2^{-l p r_{1}} \widetilde{S}_{l}^{p}\right)^{\frac{1}{p}} \\
& \ll\left(2^{n} n^{\nu-1}\right)^{\frac{1}{p}-\frac{1}{\theta}}\left(\sum_{l=n}^{\left[n_{0}\right]+1} 2^{-p l\left(r_{1}+\frac{1}{p}-\frac{1}{\theta}\right)} \widetilde{S}_{l}^{\theta}\right)^{\frac{1}{p}}
\end{aligned}
$$

Рассмотрим два случая.

Пусть $r_{1} \geqslant \frac{1}{\theta}-\frac{1}{p}$. Тогда из (3.18) имеем

$$
\begin{aligned}
I_{11} & \ll\left(2^{n} n^{\nu-1}\right)^{\frac{1}{p}-\frac{1}{\theta}} 2^{-n\left(r_{1}+\frac{1}{p}-\frac{1}{\theta}\right)}\left(\sum_{l=n}^{\left[n_{0}\right]+1} \widetilde{S}_{l}^{\theta}\right)^{\frac{1}{p}} \\
& \ll 2^{-n r_{1}} n^{(\nu-1)\left(\frac{1}{p}-\frac{1}{\theta}\right)}\|f\|_{B_{p, \theta}^{r}}^{\frac{\theta}{p}} \leqslant 2^{-n r_{1}} n^{(\nu-1)\left(\frac{1}{p}-\frac{1}{\theta}\right)} \\
& \asymp M^{-r_{1}}\left(\log ^{\nu-1} M\right)^{r_{1}+\frac{1}{p}-\frac{1}{\theta}} .
\end{aligned}
$$


Если же $0<r_{1}<\frac{1}{\theta}-\frac{1}{p}$, то из (3.18) находим

$$
I_{11} \ll\left(2^{n} n^{\nu-1}\right)^{\frac{1}{p}-\frac{1}{\theta}} 2^{-n_{0}\left(r_{1}+\frac{1}{p}-\frac{1}{\theta}\right)}\left(\sum_{l=n}^{\left[n_{0}\right]+1} \widetilde{S}_{l}^{\theta}\right)^{\frac{1}{p}} \leqslant 2^{-n r_{1}} n^{-(\nu-1) r_{1}} \asymp M^{-r_{1}} .
$$

Сопоставив $(3.20),(3.19)$ и (3.16) и воспользовавшись (3.15), приходим к искомой оценке. Оценка сверху из формулировки теоремы полностью доказана.

При доказательстве оценки снизу будем пользоваться методом, который применялся В. Н. Темляковым при установлении оценки снизу величины $e_{M}\left(W_{p, \alpha}^{r}\right)_{q}$, $1<p \leqslant q \leqslant 2$ (см., например, [3, с. 94]). Мы также сначала рассмотрим случай $1<p \leqslant q \leqslant 2$.

Итак, по заданному $M$ подберем $n \in \mathbb{N}$ таким, чтобы выполнялись соотношения $M \asymp 2^{n} n^{d-1}, 2^{n} n^{d-1} \geqslant 4 M$, и рассмотрим функцию

$$
\begin{aligned}
f_{4}(x) & =C_{8} 2^{-n\left(r_{1}+1-\frac{1}{p}\right)} n^{-\frac{d-1}{\theta}} \sum_{(s, 1)=n} \sum_{k \in \rho(s)} e^{i(k, x)} \\
& \stackrel{\mathrm{df}}{=} C_{8} 2^{-n\left(r_{1}+1-\frac{1}{p}\right)} n^{-\frac{d-1}{\theta}} \tilde{d}_{n}(x), \quad C_{8}>0 .
\end{aligned}
$$

Нетрудно проверить, что $f_{4} \in B_{p, \theta}^{r}$. Действительно,

$$
\begin{aligned}
\left\|f_{4}\right\|_{B_{p, \theta}^{r}} & =\left(\sum_{(s, 1)=n} 2^{(s, r) \theta}\left\|\delta_{s}\left(f_{4}, x\right)\right\|_{p}^{\theta}\right)^{\frac{1}{\theta}} \\
& \asymp 2^{-n\left(r_{1}+1-\frac{1}{p}\right)} n^{-\frac{d-1}{\theta}}\left(\sum_{(s, 1)=n} 2^{(s, r) \theta}\left\|\delta_{s}\left(\tilde{d}_{n}, x\right)\right\|_{p}^{\theta}\right)^{\frac{1}{\theta}} \\
& =2^{-n\left(1-\frac{1}{p}\right)} n^{-\frac{d-1}{\theta}}\left(\sum_{(s, 1)=n}\left\|\delta_{s}\left(\tilde{d}_{n}, x\right)\right\|_{p}^{\theta}\right)^{\frac{1}{\theta}} \\
& \asymp 2^{-n\left(1-\frac{1}{p}\right)} n^{-\frac{d-1}{\theta}}\left(\sum_{(s, 1)=n} 2^{(s, 1)\left(1-\frac{1}{p}\right) \theta}\right)^{\frac{1}{\theta}}=n^{-\frac{d-1}{\theta}}\left(\sum_{(s, 1)=n} 1\right)^{\frac{1}{\theta}} \ll 1 .
\end{aligned}
$$

Далее, пусть по-прежнему $\Omega_{M}$ обозначает некоторое множество из $M d$-мерных векторов $\left\{k^{1}, \ldots, k^{M}\right\}$ с целочисленными координатами. Для каждого вектора $s,(s, 1)=n$, рассмотрим множества $\Omega_{M} \cap \rho(s)$. Тогда в силу выбора числа $n$ множество $S$ векторов $s$ таких, что $(s, 1)=n$ и $\left|\Omega_{M} \cap \rho(s)\right| \leqslant \frac{1}{2}|\rho(s)|$, будет содержать по-крайней мере половину всех $s$ таких, что $\|s\|_{1}=n$, и, следовательно, $|S| \asymp n^{d-1}$. Пусть далее $t(x)$ обозначает произвольный полином с "номерами" 
гармоник из $\Omega_{M}$. Тогда согласно лемме Г будем иметь

$$
\begin{aligned}
\left\|f_{4}-t\right\|_{q} & \gg\left(\sum_{(s, 1)=n}\left\|\delta_{s}\left(f_{4}-t, x\right)\right\|_{2}^{q} 2^{\|s\|_{1}\left(\frac{1}{2}-\frac{1}{q}\right)_{q}}\right)^{\frac{1}{q}} \\
& \gg\left(\sum_{s \in S}\left\|\delta_{s}\left(f_{4}-t, x\right)\right\|_{2}^{q}\right)^{\frac{1}{q}} 2^{n\left(\frac{1}{2}-\frac{1}{q}\right)} \\
& \gg|S|^{\frac{1}{q}} 2^{-n\left(r_{1}+1-\frac{1}{p}\right)} n^{-\frac{d-1}{\theta}} 2^{n\left(\frac{1}{2}-\frac{1}{q}\right)} 2^{\frac{n}{2}} \\
& =2^{-n\left(r_{1}-\frac{1}{p}+\frac{1}{q}\right)} n^{(d-1)\left(\frac{1}{q}-\frac{1}{\theta}\right)} \\
& \asymp M^{-\left(r_{1}-\frac{1}{p}+\frac{1}{q}\right)}\left(\log ^{d-1} M\right)^{r_{1}-\frac{1}{p}+\frac{2}{q}-\frac{1}{\theta}}
\end{aligned}
$$

Полученная оценка совпадает по порядку с оценкой сверху $e_{M}\left(B_{p, \theta}^{r}\right)_{q}$ в случае $r_{1}>\frac{1}{p}-\frac{2}{q}+\frac{1}{\theta}$. Если же $\frac{1}{p}-\frac{1}{q}<r_{1} \leqslant \frac{1}{p}-\frac{2}{q}+\frac{1}{\theta}$, то необходимая оценка снизу следует из одномерного случая.

В завершение осталось рассмотреть случай $p=1,1<q \leqslant 2$. Пусть числа $M$ и $n$ имеют прежний смысл. Рассмотрим функцию

$$
f_{5}(x)=C_{9} n^{-\frac{d-1}{\theta}} \sum_{(s, 1)=n} \sum_{k \in \rho^{+}(s)} \prod_{j=1}^{d} k_{j}^{-r_{1}} \cos k_{j} x_{j}, \quad C_{9}>0 .
$$

Аналогично тому, как было показано вьше для функции $f_{2}(x)$ (см. $\left.(2.54)\right)$, нетрудно проверить, что и функция $f_{5}(x)$ принадлежит классу $B_{1, \theta}^{r}$. Тогда в силу леммы Г будем иметь

$$
\begin{aligned}
\left\|f_{5}-t\right\|_{q} & \gg\left(\sum_{(s, 1)=n}\left\|\delta_{s}\left(f_{5}-t, x\right)\right\|_{2}^{q} 2^{\|s\|_{1}\left(\frac{1}{2}-\frac{1}{q}\right) q}\right)^{\frac{1}{q}} \\
& \gg\left(\sum_{s \in S}\left\|\delta_{s}\left(f_{5}-t, x\right)\right\|_{2}^{q} 2^{\|s\|_{1}\left(\frac{1}{2}-\frac{1}{q}\right) q}\right)^{\frac{1}{q}} .
\end{aligned}
$$

Далее, поскольку для каждого $s \in S$

$$
\left\|\delta_{s}\left(f_{5}-t, x\right)\right\|_{2} \gg n^{-\frac{d-1}{\theta}} \prod_{j=1}^{d} 2^{-s_{j} r_{1}} 2^{\frac{s_{j}}{2}}=2^{-(s, 1)\left(r_{1}-\frac{1}{2}\right)} n^{-\frac{d-1}{\theta}},
$$

то из (3.21) находим

$$
\begin{aligned}
\left\|f_{5}-t\right\|_{q} & \gg\left(\sum_{s \in S} 2^{-\|s\|_{1}\left(r_{1}-1+\frac{1}{q}\right) q}\right)^{\frac{1}{q}} n^{-\frac{d-1}{\theta}} \\
& \asymp 2^{-n\left(r_{1}-1+\frac{1}{q}\right)}\left(\sum_{s \in S} 1\right)^{\frac{1}{q}} n^{-\frac{d-1}{\theta}} \asymp 2^{-n\left(r_{1}-1+\frac{1}{q}\right)} n^{(d-1)\left(\frac{1}{q}-\frac{1}{\theta}\right)} \\
& \asymp M^{-\left(r_{1}-1+\frac{1}{q}\right)}\left(\log ^{d-1} M\right)^{r_{1}-1+\frac{2}{q}-\frac{1}{\theta}} .
\end{aligned}
$$


Оценки снизу, а вместе с ними и теорема доказаны.

Отметим следуюшее. Точные порядки величин $e_{M}\left(W_{p, \alpha}^{r}\right)_{q}, 1<p \leqslant q \leqslant 2$, $r_{1}>2\left(\frac{1}{p}-\frac{1}{q}\right)$, и $e_{M}\left(H_{p}^{r}\right)_{q}, 1<p \leqslant q \leqslant 2, r_{1}>\frac{1}{p}-\frac{1}{q}$, были установлены В. Н. Темляковым (см., например, [3, с. 92]). Затем Э. С. Белинский [16] получил оценку сверху величины $e_{M}\left(W_{p, \alpha}^{r}\right)_{q}, 1<p \leqslant q \leqslant 2$, и в случае $\frac{1}{p}-\frac{1}{q}<r_{1} \leqslant 2\left(\frac{1}{p}-\frac{1}{q}\right)$, которая в сочетании с оценкой снизу, установленной ранее В. Н. Темляковым, дала возможность получить точный порядок $e_{M}\left(W_{p, \alpha}^{r}\right)_{q}, 1<p \leqslant q \leqslant 2$, для всех $r_{1}>\frac{1}{p}-\frac{1}{q}$. Порядки величин $e_{M}\left(W_{1, \alpha}^{r}\right)_{q}$ и $e_{M}\left(H_{1}^{r}\right)_{q}, 1<q \leqslant 2$, следуют из соответствующих оценок $M$-членных приближений ядер Бернулли $F_{r}(x, \alpha)$ (см. [14]) и оценок приближения классов $H_{1}^{r}$ ступенчатыми гиперболическими суммами $\Phi$ рье $[3$, с. 35$]$.

Tеорема 3.2. Пусть $1<q \leqslant p<\infty, \quad p \geqslant 2, r_{1}>0$. Тогда при $1 \leqslant \theta \leqslant \infty$ справедлива оиенка

$$
e_{M}\left(B_{p, \theta}^{r}\right)_{q} \asymp M^{-r_{1}}\left(\log ^{\nu-1} M\right)^{\left(r_{1}+\frac{1}{2}-\frac{1}{\theta}\right)_{+}} .
$$

ДокАЗАТЕЛЬство. Поскольку $\|\cdot\|_{q} \leqslant\|\cdot\|_{p}$ при $q \leqslant p$, то оценка сверху величины $e_{M}\left(B_{p, \theta}^{r}\right)_{q}$ сводится к оценке сверху $e_{M}\left(B_{p, \theta}^{r}\right)_{p}, 2 \leqslant p<\infty$.

Пусть $f \in B_{p, \theta}^{r}$ и $2<\theta<\infty$. Тогда в силу теоремы Литтлвуда-Пэли, неравенства Минковского и неравенства Гёльдера будем иметь при $M \asymp 2^{n} n^{d-1}$

$$
\begin{aligned}
& e_{M}(f)_{p} \ll\left\|f(x)-S_{n}^{\gamma^{\prime}}(f, x)\right\|_{p}=\left\|\sum_{\left(s, \gamma^{\prime}\right) \geqslant n} \delta_{s}(f, x)\right\|_{p} \\
& \ll\left\|\left(\sum_{\left(s, \gamma^{\prime}\right) \geqslant n}\left|\delta_{s}(f, x)\right|^{2}\right)^{\frac{1}{2}}\right\|_{p}=\left(\left\|\sum_{\left(s, \gamma^{\prime}\right) \geqslant n}\left|\delta_{s}(f, x)\right|^{2}\right\|_{\frac{p}{2}}\right)^{\frac{1}{2}} \\
& \leqslant\left(\sum_{\left(s, \gamma^{\prime}\right) \geqslant n}\left\|\delta_{s}(f, x)\right\|_{p}^{2}\right)^{\frac{1}{2}} \\
& \leqslant\left(\sum_{\left(s, \gamma^{\prime}\right) \geqslant n} 2^{2(s, r) \theta}\left\|\delta_{s}(f, x)\right\|_{p}^{\theta}\right)^{\frac{1}{\theta}}\left(\sum_{\left(s, \gamma^{\prime}\right) \geqslant n} 2^{-2(s, r) \frac{\theta}{\theta-2}}\right)^{\frac{1}{2}-\frac{1}{\theta}} \\
& \ll\|f\|_{B_{p, \theta}^{r}}\left(\sum_{\left(s, \gamma^{\prime}\right) \geqslant n} 2^{-2(s, r) \frac{\theta}{\theta-2}}\right)^{\frac{1}{2}-\frac{1}{\theta}} \\
& \leqslant\left(\sum_{\left(s, \gamma^{\prime}\right) \geqslant n} 2^{-2 r_{1}(s, \gamma) \frac{\theta}{\theta-2}}\right)^{\frac{1}{2}-\frac{1}{\theta}} \ll 2^{-n r_{1}} n^{(\nu-1)\left(\frac{1}{2}-\frac{1}{\theta}\right)} \\
& \asymp M^{-r_{1}}\left(\log ^{\nu-1} M\right)^{r_{1}+\frac{1}{2}-\frac{1}{\theta}} \text {. }
\end{aligned}
$$

Также нетрудно убедиться в том, что при $\theta=2$

$$
e_{M}(f)_{p} \ll M^{-r_{1}}\left(\log ^{\nu-1} M\right)^{r_{1}} .
$$


В случае $\theta=\infty$, учитьвая, что $\left\|\delta_{s}(f, x)\right\|_{p} \ll 2^{-(s, r)}$, получим

$$
\begin{aligned}
e_{M}(f)_{q} & \leqslant\left\|f(x)-S_{n}^{\gamma^{\prime}}(f, x)\right\|_{p} \ll\left(\sum_{\left(s, \gamma^{\prime}\right) \geqslant n}\left\|\delta_{s}(f, x)\right\|_{p}^{2}\right)^{\frac{1}{2}} \\
& \ll\left(\sum_{\left(s, \gamma^{\prime}\right) \geqslant n} 2^{-2(s, r)}\right)^{\frac{1}{2}} \ll 2^{-n r_{1}} n^{\frac{\nu-1}{2}} \asymp M^{-r_{1}}\left(\log ^{\nu-1} M\right)^{r_{1}+\frac{1}{2}} .
\end{aligned}
$$

Таким образом, из (3.22), $\left(3.22^{\prime}\right)$ и (3.23) получаем требуемые оценки сверху величины $e_{M}\left(B_{p, \theta}^{r}\right)_{p}$ в случае $2 \leqslant \theta \leqslant \infty$.

Пусть теперь $1 \leqslant \theta<2$. Тогда, проведя рассуждения, аналогичные изложенным выше для случая $1<p=q \leqslant 2,1 \leqslant \theta<p$, получим следуюшие оценки для $I_{10}$ и $I_{11}:$

$$
\begin{aligned}
& I_{10} \ll M^{-r_{1}}, \\
& I_{11} \ll M^{-r_{1}}\left(\log ^{\nu-1} M\right)^{\left(r_{1}+\frac{1}{2}-\frac{1}{\theta}\right)_{+}} .
\end{aligned}
$$

Воспользовавшись соотношением (3.18), из (3.24) и (3.25) приходим к искомой оценке и в случае $1 \leqslant \theta<2$.

При доказательстве оценки снизу мы будем использовать схему рассуждений, которая применялась Б. С. Кашиньм и В. Н. Темляковым в [18] при нахождении оценки снизу величины $e_{M}\left(W_{p, \alpha}^{r}\right)_{q}, 1<q<p<\infty, p \geqslant 2$. Заметим также, что при этом достаточно ограничиться случаем $1<q<2$.

Приведем необходимые обозначения и определения.

Для вектора $s=\left(s_{1}, \ldots, s_{d}\right), s_{j}$ - четные числа, $s_{j}>0, j=\overline{1, d}$, по-прежнему будем обозначать

$$
\rho^{+}(s)=\left\{k: 2^{s_{j}-1} \leqslant k_{j}<2^{s_{j}}, \quad k_{j} \in \mathbb{N}\right\}
$$

и для $n \in \mathbb{N}$ положим

$$
B_{n}=\left\{s:(s, 1)=2\left[\frac{n}{2}\right]\right\}, \quad \bar{Q}_{n}^{\prime}=\bigcup_{s \in B_{n}} \rho^{+}(s) .
$$

Пусть $\mathscr{T}\left(\bar{Q}_{n}^{\prime}\right)$ - множество полиномов вида $t(x)=\sum_{|k| \in \bar{Q}_{n}^{\prime}} c_{k} e^{i(k, x)}$, где $|k|=$ $\left(\left|k_{1}\right|, \ldots,\left|k_{d}\right|\right)$. Наряду с нормой пространства $L_{q}$ будем рассматривать норму пространства $B_{q, \theta}^{0}$, которую для тригонометрических полиномов $t(x) \in \mathscr{T}\left(\bar{Q}_{n}^{\prime}\right)$ определим формулой

$$
\|t\|_{B_{q, \theta}^{0}}=\left(\sum_{s \in B_{n}}\left\|A_{s}(t, x)\right\|_{q}^{\theta}\right)^{1 / \theta}, \quad 1 \leqslant q \leqslant \infty, \quad 1 \leqslant \theta \leqslant \infty,
$$

с естественной модификацией определения при $\theta=\infty$. Аналогичным образом определяется $\|f\|_{B_{q, \theta}^{0}}$ для функций $f(x)$ из $L_{q}\left(\pi_{d}\right)$ при условии сходимости ряда $\sum_{s}\left\|A_{s}(f, x)\right\|_{q}^{\theta}$. Отметим, что если $1<q<\infty$, то

$$
\|f\|_{B_{q, \theta}^{0}} \asymp\left(\sum_{s}\left\|\delta_{s}(f, x)\right\|_{q}^{\theta}\right)^{1 / \theta} .
$$


Пусть $\mathscr{D}$ - некоторая ограниченная область в $\mathbb{R}^{d}$ и $\Phi=\left\{\varphi_{n}(x)\right\}_{n=1}^{\infty}-$ система функций из пространства $L_{q}(\mathscr{D})$. Для $f \in L_{q}(\mathscr{D})$ положим

$$
e_{M}(f, \Phi)_{q}=\inf _{\substack{\left\{n_{i}\right\}=\Lambda \subset \mathbb{Z}_{+},|\Lambda|=M \\\left\{c_{i}\right\} \in \mathbb{R}^{M}}}\left\|f(x)-\sum_{i=1}^{M} c_{i} \varphi_{n_{i}}(x)\right\|_{L_{q}(\mathscr{D})}
$$

Если $F$ - некоторый класс функций из $L_{q}(\mathscr{D})$, то полагаем

$$
e_{M}(F, \Phi)_{q}=\sup _{f \in F} e_{M}(f, \Phi)_{q}
$$

Справедливо следующее утверждение.

Tеорема $\Gamma$ [18]. Существует постоянная $C(d)>0$ такая, что для любого набора функций $\Phi=\left\{\varphi_{j}\right\}_{j=1}^{l} \subset B_{1,1}^{0}, \quad l<C^{\prime}\left|\bar{Q}_{n}^{\prime}\right|$, справедлива оченка

$$
e_{M}\left(\mathscr{T}\left(\bar{Q}_{n}^{\prime}\right)_{B_{\infty, \infty}^{0}}, \Phi\right)_{B_{1,1}^{0}} \geqslant C_{1} n^{d-1}, \quad C_{1}=C_{1}\left(d, C^{\prime}\right)>0
$$

для всех $M \leqslant C(d)\left|\bar{Q}_{n}^{\prime}\right|$.

Теперь перейдем непосредственно к установлению оценки снизу в нашей теореме. По заданному $M$ подберем $n$ из соотношения $M \asymp 2^{n} n^{d-1}$, и пусть $P_{\bar{Q}_{n}^{\prime}}$ - оператор ортогонального проектирования на $\mathscr{T}\left(\bar{Q}_{n}^{\prime}\right)$. Как следует из теоремы Литтлвуда-Пэли (см., например, [3, с. 7]), этот оператор ограничен как оператор, действуюший из $L_{q}$ в $L_{q}$ при $q \in(1, \infty)$, и, таким образом, для тригонометрической системы $T=\left\{e^{i(k, x)}\right\}_{k \in \mathbb{Z}^{d}}$ справедливо соотношение

$$
e_{M}\left(\mathscr{T}\left(\bar{Q}_{n}^{\prime}\right)_{B_{\infty, \infty}^{0}}, T\right)_{q} \gg e_{M}\left(\mathscr{T}\left(\bar{Q}_{n}^{\prime}\right)_{B_{\infty, \infty}^{0}},\left\{e^{i(k, x)}\right\}_{k \in \bar{Q}_{n}^{\prime}}\right)_{q}
$$

Далее, поскольку для $t(x) \in \mathscr{T}\left(\bar{Q}_{n}^{\prime}\right)_{B_{\infty, \infty}^{0}}$

$$
\begin{aligned}
\|t\|_{B_{\infty, \theta}^{r}} & =\left(\sum_{s \in B_{n}} 2^{(s, r) \theta}\left\|A_{s}(t, x)\right\|_{\infty}^{\theta}\right)^{\frac{1}{\theta}} \\
& \leqslant 2^{n r_{1}} \max _{s \in B_{n}}\left\|A_{s}(t, x)\right\|_{\infty}\left(\sum_{s \in B_{n}} 1\right)^{\frac{1}{\theta}} \ll 2^{n r_{1}}\|t\|_{B_{\infty, \infty}^{0}} n^{\frac{d-1}{\theta}}
\end{aligned}
$$

то отсюда заключаем, что сушествует постоянная $C_{10}>0$ такая, что

$$
C_{10} 2^{-n r_{1}} n^{-\frac{d-1}{\theta}} \mathscr{T}\left(\bar{Q}_{n}^{\prime}\right)_{B_{\infty, \infty}^{0}} \subset B_{\infty, \theta}^{r} \cap \mathscr{T}\left(\bar{Q}_{n}^{\prime}\right)
$$


Из (3.26) и (3.27) находим

$$
\begin{aligned}
e_{M}\left(B_{\infty, \theta}^{r}\right)_{q} & \geqslant e_{M}\left(B_{\infty, \theta}^{r} \cap \mathscr{T}\left(\bar{Q}_{n}^{\prime}\right)\right)_{q} \\
& \gg 2^{-n r_{1}} n^{-\frac{d-1}{\theta}} e_{M}\left(\mathscr{T}\left(\bar{Q}_{n}^{\prime}\right)_{B_{\infty, \infty}^{0}},\left\{e^{i(k, x)}\right\}_{\left.k \in \bar{Q}_{n}^{\prime}\right)_{q}}\right.
\end{aligned}
$$

Теперь запишем соотношение между нормами полиномов из $\mathscr{T}\left(\bar{Q}_{n}^{\prime}\right)$ в пространствах $L_{q}$ при $1<q \leqslant 2$ и $B_{1,1}^{0}$. С одной стороны, для $t \in \mathscr{T}\left(\bar{Q}_{n}^{\prime}\right)$ (см. [18])

$$
\|t\|_{q} \gg\left(\sum_{s \in B_{n}}\left\|\delta_{s}(t, x)\right\|_{q}^{q}\right)^{\frac{1}{q}} \gg\|t\|_{B_{q, 2}^{0}},
$$

а с другой стороны,

$$
\begin{aligned}
\|t\|_{B_{1,1}^{0}} & \asymp \sum_{s \in B_{n}}\left\|A_{s}(t, x)\right\|_{1} \leqslant\left(\sum_{s \in B_{n}}\left\|A_{s}(t, x)\right\|_{1}^{2}\right)^{\frac{1}{2}}\left(\sum_{s \in B_{n}} 1\right)^{\frac{1}{2}} \\
& \ll\|t\|_{B_{1,2}^{0}} n^{\frac{d-1}{2}} \leqslant\|t\|_{B_{q, 2}^{0}} n^{\frac{d-1}{2}} .
\end{aligned}
$$

Сопоставив $(3.29)$ и $(3.30)$, получаем

$$
\|t\|_{q} \gg n^{-\frac{d-1}{2}}\|t\|_{B_{1,1}^{0}}
$$

Таким образом, согласно (3.31) из (3.28) приходим к оценке

$$
e_{M}\left(B_{\infty, \theta}^{r}\right)_{q} \gg 2^{-n r_{1}} n^{-(d-1)\left(\frac{1}{2}+\frac{1}{\theta}\right)} e_{M}\left(\mathscr{T}\left(\bar{Q}_{n}^{\prime}\right)_{B_{\infty, \infty}^{0}},\left\{e^{i(k, x)}\right\}_{k \in \bar{Q}_{n}^{\prime}}\right)_{B_{1,1}^{0}}
$$

Применив к (3.32) теорему Г при $\Phi=\left\{e^{i(k, x)}\right\}_{k \in \bar{Q}_{n}^{\prime}}$ и $l=\left|\bar{Q}_{n}^{\prime}\right|$, находим

$$
e_{M}\left(B_{\infty, \theta}^{r}\right)_{q} \gg 2^{-n r_{1}} n^{(d-1)\left(\frac{1}{2}-\frac{1}{\theta}\right)} \asymp M^{-r_{1}}\left(\log ^{d-1} M\right)^{r_{1}+\frac{1}{2}-\frac{1}{\theta}} .
$$

Наконец, принимая во внимание, что $B_{\infty, \theta}^{r} \subset B_{p, \theta}^{r}$ при $1 \leqslant p<\infty$, из (3.33) получаем оценку

$$
e_{M}\left(B_{p, \theta}^{r}\right)_{q} \gg M^{-r_{1}}\left(\log ^{d-1} M\right)^{r_{1}+\frac{1}{2}-\frac{1}{\theta}},
$$

которая совпадает по порядку с оценкой сверху в случае $r_{1} \geqslant \frac{1}{\theta}-\frac{1}{2}$. Если же $0<r_{1}<\frac{1}{\theta}-\frac{1}{2}$, то искомая оценка следует из одномерного случая. Теорема доказана.

В заключение отметим, что порядки величин $e_{M}\left(W_{p, \alpha}^{r}\right)_{q}$ и $e_{M}\left(H_{p}^{r}\right)_{q}, 1<q<$ $p<\infty, p \geqslant 2$, известны. Оценки снизу этих величин установлены в [18, с. 74]. Соответствуюшие оценки сверху являются следствием оценок приближения классов $W_{p, \alpha}^{r}$ и $H_{p}^{r}$ ступенчатыми гиперболическими суммами Фурье, которые получены соответственно в [36] и [37].

Обратим также внимание, что величины $e_{M}\left(B_{p, \theta}^{r}\right)_{q}, 1<q \leqslant p<\infty, p \geqslant 2$, в случае $0<r_{1}<\frac{1}{\theta}-\frac{1}{2}$ и $\nu>1$ убывают при $M \rightarrow \infty$ сушественно быстрее наилучших приближений классов $B_{p, \theta}^{r}$ тригонометрическими полиномами с "номерами" гармоник из соответствуюших гиперболических крестов [38]. 


\section{Список литературы}

1. Бесов О.В. О некотором семействе функциональных пространств. Теоремы вложения и продолжения // ДАН СССР. 1959. Т. 126. №6. С. 1163-1165.

2. Лизоркин П.И., Никольский С. М. Пространства функций смешанной гладкости с декомпозиционной точки зрения // Тр. Матем. ин-та им. В. А. Стеклова АН СССР. 1989. T. 187. C. $143-161$.

3. Темляков В. Н. Приближение функций с ограниченной смешанной производной // Тр. Матем. ин-та им. В.А. Стеклова АН СССР. 1986. Т. 178. С. 1-112.

4. Никольский C. М. Приближение функций многих переменных и теоремы вложения. М.: Наука, 1969.

5. Стечкин С. Б. Об абсолютной сходимости ортогональных рядов // ДАН СССР. 1955. T. 102. № 2. C. $37-40$.

6. Исмагилов Р. С. Поперечники множеств в линейных нормированных пространствах и приближение функций тригонометрическими многочленами // УМН. 1974. Т. 29. №3. C. $161-178$.

7. Осколков К.И. Аппроксимационные свойства суммируемых функций на множествах полной меры // Матем. сб. 1977. Т. 103. № 4. С. 563-589.

8. Майоров В. Е. О линейных поперечниках соболевских классов // ДАН СССР. 1978. T. 243. № 5. C. 1127-1130.

9. Майоров B.E. О линейных поперечниках соболевских классов и цепочках экстремальных подпространств // Матем. сб. 1980. Т. 113. № 3. С. 437-463.

10. Macovoz G. Y. On trigonometric n-widths and their generalization // J. of Approxim. Theory. 1984. V. 41. № 4. P. 361-366.

11. Кашин Б. С. Об аппроксимационных свойствах полных ортонормированных систем // Тр. Матем. ин-та им. В.А. Стеклова АН СССР. 1985. Т. 172. С. 187-191.

12. Белинский Э.С. Приближение "плавающей" системой экспонент на классах гладких периодических функций // Матем. сб. 1987. Т. 132. № 1. С. 20-27.

13. Темляков В.Н. О приближении периодических функций многих переменных // ДАН CCCP. 1984. T. 279. № 2. C. 301-305.

14. Белинский Э. С. Приближение периодических функций многих переменных "плавающей" системой экспонент и тригонометрические поперечники // ДАН СССР. 1985. T. 284. №6. C. 1294-1297.

15. Белинский Э. С. Приближение “плавающей” системой экспонент на классах периодических гладких функций // Тр. Матем. ин-та им. В. А. Стеклова АН СССР. 1987. Т. 180. C. $46-47$.

16. Белинский Э. С. Приближение “плавающей” системой экспонент на классах периодических функций с ограниченной смешанной производной // Исследования по теории функций многих вещественных переменных. Ярославль: Ярославский гос. ун-т, 1988. C. $16-33$.

17. Belinskii E. S. Approximation of functions of several variables by trigonometric polynomials with given number of harmonics, and estimates of $\varepsilon$-entropy // Anal. Math. 1989. V. 15. № 2. P. $67-74$.

18. Кашин Б. С., Темляков В.Н. О наилучших $m$-членных приближениях и энтропии множеств в пространстве $L_{1} / /$ Матем. заметки. 1994. Т. 56. № 5. С. 57-86.

19. Темляков В.Н. Нелинейные поперечники по Колмогорову // Матем. заметки. 1998. T. 63. №6. C. 891-902.

20. De Vore R. A., Temlyakov V. N. Nonlinear Approximation in Finite-Dimensional Spaces // J. of complexity. 1997. V. 13. P. 489-508.

21. Temlyakov V. N. Greedy Algorithm and $m$-Term Trigonometric Approximation // Constructive Approx. 1998. V. 14. P. 569-587. 
22. Temlyakov V.N. Greedy Algorithms and $m$-Term Approximation with Regard to Redundant Dictionaries // J. of Approximation Theory. 1999. V. 98. P. 117-145.

23. Dinh Dung. On Asymptotic Orders of $n$-Term Approximations and Non-Linear $n$-Widths // Vietnam J. of Mathematics. 1999. V. 27. № 4. P. 363-367.

24. Dinh Dung. Continuons Algorithms in $n$-Term Approximation and Non-Linear-Widths // J. of Approximation Theory. 2000. V. 102. P. 217-242.

25. Belinskii E. S. Decomposition theorems and approximation by a "floating" system of exponentials // Transactions of the American Mathematical Society. 1998. V. 350. № 1 . P. $43-53$.

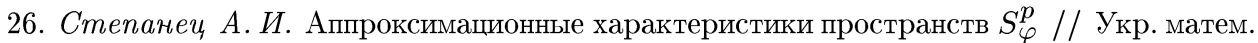
журн. 2001. Т. 53. №3. С. 392-416.

27. Романюк А. С. О приближении классов Бесова периодических функций многих переменных // Межд. конф. "Функциональные пространства, теория приближений, нелинейный анализ”, посвященная 90-летию акад. С. М. Никольского. Москва, 27 апреля - 3 мая 1995. C. 231-232.

28. Романюк A.C. О наилучшей тригонометрической и билинейной аппроксимации классов Бесова функций многих переменных // Укр. матем. журн. 1995. Т. 47. № 8. С. 1097-1111.

29. Романюк A. С. Об оценках аппроксимативных характеристик классов Бесова периодических функций многих переменных // Укр. матем. журн. 1997. Т. 49. № 9. С. 1250-1261.

30. Никольский C.M. Неравенства для целых функций конечной степени и их применение в теории дифференцируемых функций многих переменных // Тр. Матем. ин-та им. В. А. Стеклова АН СССР. 1951. Т. 38. С. 244-278.

31. Jakson D. Certain problem of closest approximation // Bull. Amer. Math. Soc. 1933. V. 39. P. 889-906.

32. Кашин Б. С. О поперечниках классов Соболева малой гладкости // Вестн. Моск. ун-та. Мат. и мех. 1981. № 5. С. 50-54.

33. Харди Г., Литтлвуд Д., Полиа Г. Неравенства. М.: ИЛ, 1948.

34. Корнейчук Н. П. Точные константы в теории приближения. М.: Наука, 1987.

35. Кашин Б. С., Саакян А. А. Ортогональные ряды. М.: Наука, 1984.

36. Галеев Э. М. Приближение некоторых классов периодических функций многих переменных суммами Фурье в метрике $L_{p}$ // УМН. 1977. Т. 32. № 3. С. 251-252.

37. Никольская Н. С. Приближение периодических функций класса $S H_{p^{*}}^{r}$ суммами Фурье // Сиб. матем. журн. 1975. Т. 16. № 4. С. 761-780.

38. Романюк A.C. Приближение классов периодических функций многих переменных в пространстве $L_{q} / /$ Укр. матем. журн. 1991. Т. 43. № 10. С. 1398-1408.

Институт математики НАН Украины

Поступило в редакцию 27.VII.2001 